\title{
Supporting Information for \\ Rational Design of an L-Histidine-derived Minimal Artificial Acylase for the Kinetic Resolution of Racemic Alcohols
}

\author{
Kazuaki Ishihara, ${ }^{*}{ }^{\dagger}$ Yuji Kosugi ${ }^{\dagger}$ and Matsujiro Akakura ${ }^{\ddagger}$
}

Graduate School of Engineering, Nagoya University, Furo-cho, Chikusa, Nagoya 464-8603, Japan, and Department of Chemistry, Aichi University of Education, Igaya-cho, Kariya 448-8542, Japan

General Methods. Infrared (IR) spectra were recorded on a JASCO FT/IR 460 plus spectrometer. ${ }^{1} \mathrm{H}$ NMR spectra were measured on a Varian Gemini-2000 spectrometer (300 MHz) at ambient temperature. Data were recorded as follows: chemical shift in ppm from internal tetramethylsilane on the $\delta$ scale, multiplicity ( $\mathrm{s}=$ singlet; $\mathrm{d}=$ doublet; $\mathrm{t}=$ triplet; $\mathrm{m}=$ multiplet), coupling constant $(\mathrm{Hz})$, integration, and assignment. ${ }^{13} \mathrm{C}$ NMR spectra were measured on Varian Gemini-2000 (75 MHz) spectrometer. Chemical shifts were recorded in ppm from the solvent resonance employed as the internal standard (deuterochloroform at $77.00 \mathrm{ppm}$ ). Highperformance liquid chromatography (HPLC) analysis was conducted using Shimadzu LC-10 AD coupled diode array-detector SPD-MA-10A-VP and chiral column of Daicel CHIRALCEL OD-H $(4.6 \mathrm{~mm} \times 25 \mathrm{~cm})$, AD-H $(4.6 \mathrm{~mm} \times 25 \mathrm{~cm})$, or Daicel CHIRALPAK AS-H $(4.6 \mathrm{~mm} \times 25 \mathrm{~cm})$. Optical rotations were measured on a RUDOLPH AUTOPOL IV digital polarimeter. GC analysis was performed with Shimadzu 17A instruments using TCI CHIRALDEX $\gamma$-TA (0.25 mmI.D. x 20 $\mathrm{m} \times 0.125 \mu \mathrm{m})$. Melting points were determined using a Yanaco MP-J3. All experiments were carried out under an atmosphere of dry nitrogen. For thin-layer chromatography (TLC) analysis throughout this work, Merck precoated TLC plates (silica gel $60 \mathrm{GF}_{254} 0.25 \mathrm{~mm}$ or silica gel $\mathrm{NH}_{2}$ $\mathrm{F}_{254 \mathrm{~S}} 0.25 \mathrm{~mm}$ ) were used. The products were purified by column chromatography on silica gel (E. Merck Art. 9385 or Fuji Silysia Chemical Ltd. Cromatorex ${ }^{\circledR}$ NH-DM1020). Microanalyses were performed at the Graduate School of Agriculture, Nagoya University. High resolution mass spectral analysis (HRMS) was performed at Chemical Instrument Center, Nagoya University. In experiments that required dry solvent, ether, $N, N$-dimethylformamide (DMF) and tetrahydorofuran (THF) were purchased from Aldrich or Wako as the "anhydrous" and stored over 4A molecular sieves. Benzene, hexane, toluene, and dichloromethane were freshly distilled from calcium hydride. Other simple chemicals were analytical-grade and obtained commercially.

General Procedure for the Preparation of $N(\pi)$-Methyl- $N(\alpha)$-(arenesulfonyl)-L-hisitidinol

Nagoya University.

Aichi University of Education. 
(1, $\mathbf{X}=\mathbf{O H})$. To a solution of $N(\pi)$-methyl-L-hisitidinol $(3)^{1}(621 \mathrm{mg}, 4.0 \mathrm{mmol})$ in pyridine $(20$ $\mathrm{mL}$ ) was added the corresponding arenesulfonyl chloride $(5.5 \mathrm{mmol})$ at $0{ }^{\circ} \mathrm{C}$. After the mixture was stirred for $5 \mathrm{~h}$ at room temperature, the solvent was removed under reduced pressure. The crude product was dissolved in EtOAc, and washed with water and brine. The organic layer was dried over $\mathrm{Na}_{2} \mathrm{SO}_{4}$, filtrated, and concentrated under reduced pressure. The reside was purified by flah column chromatography on Cromatorex ${ }^{\circledR}$ NH-DM1020 (eluent: EtOAc) to give $N(\pi)$-methyl$N(\alpha)$-(arenesulfonyl)-L-hisitidinol $(\mathbf{1}, \mathrm{X}=\mathrm{OH})$ in good yield.

$N(\pi)$-Methyl- $N(\alpha)$-(benzenesulfonyl)-L-hisitidinol $\left(\mathbf{1}, \mathrm{Ar}=\mathrm{C}_{6} \mathbf{H}_{5}, \mathrm{X}=\mathbf{O H}\right): \quad$ TLC (silica gel $\mathrm{NH}_{2} \mathrm{~F}_{254 \mathrm{~S}}$, EtOAc:MeOH=11:1) $R_{\mathrm{f}}=0.25$; Purification by column chromatography on Cromatorex ${ }^{\circledR}$ NH-DM1020 (EtOAc:MeOH=10:1); $[\alpha]_{\mathrm{D}}{ }^{20}=6.0\left(c=1.06, \mathrm{CHCl}_{3}\right)$; IR $(\mathrm{KBr}) 3600-3250,2924$, 1636, 1510, 1447, 1324, 1158, 1093, 690, $592 \mathrm{~cm}^{-1}$; ${ }^{1} \mathrm{H}$ NMR $\left(300 \mathrm{MHz}, \mathrm{CDCl}_{3}\right) \delta 2.75(\mathrm{dd}, J=$ 6.0, $15.3 \mathrm{~Hz}, 1 \mathrm{H}), 2.88$ (dd, $J=7.5,15.3 \mathrm{~Hz}, 1 \mathrm{H}), 3.32-3.38(\mathrm{~m}, 1 \mathrm{H}), 3.39$ (s, 3H), 3.49 (d, $J=4.2$ $\mathrm{Hz}, 2 \mathrm{H}), 4.48-4.95(\mathrm{br}, 1 \mathrm{H}), 6.61(\mathrm{~s}, 1 \mathrm{H}), 7.26(\mathrm{~s}, 1 \mathrm{H}), 7.42(\mathrm{t}, J=7.4 \mathrm{~Hz}, 2 \mathrm{H}), 7.51$ (t, $J=7.4 \mathrm{~Hz}$, $1 \mathrm{H}), 7.77(\mathrm{~d}, J=7.4 \mathrm{~Hz}, 2 \mathrm{H}) ;{ }^{13} \mathrm{C} \mathrm{NMR}\left(75 \mathrm{MHz} \mathrm{CDCl}_{3}\right) \delta 25.9,31.4,54.3,62.3,126.7$ (2C), 127.4, 127.9, 129.0 (2C), 132.4, 137.8, 140.5. Anal. Calcd for $\mathrm{C}_{13} \mathrm{H}_{17} \mathrm{~N}_{3} \mathrm{O}_{3} \mathrm{~S}$ : C, 52.86; H, 5.80. Found: $\quad$ C, 52.81; H, 5.82.

$N(\pi)$-Methyl- $N(\alpha)$-(4-trifluoromethylbenzenesulfonyl)-L-hisitidinol $\left(1, \quad \mathrm{Ar}=4-\mathrm{CF}_{3} \mathrm{C}_{6} \mathrm{H}_{2}\right.$, $\mathbf{X}=\mathbf{O H}): \quad{ }^{1} \mathrm{H} \mathrm{NMR}\left(\mathrm{CDCl}_{3}, 300 \mathrm{MHz}\right) \delta 2.84(\mathrm{dd}, J=6.0,15.3 \mathrm{~Hz}, 1 \mathrm{H}), 2.92(\mathrm{dd}, J=7.1,15.5 \mathrm{~Hz}$, 1H), $3.41(\mathrm{~m}, 1 \mathrm{H}), 3.49$ (s, 3H), 3.54 (d, J = 4.2 Hz, 2H), 6.02 (br, 1H), 6.60 (s, 1H), 7.28 (s, 1H), $7.67(\mathrm{~d}, J=8.1 \mathrm{~Hz}, 2 \mathrm{H}), 7.93(\mathrm{~d}, J=8.4 \mathrm{~Hz}, 2 \mathrm{H})$. Anal. Calcd for $\mathrm{C}_{14} \mathrm{H}_{16} \mathrm{~F}_{3} \mathrm{~N}_{3} \mathrm{O}_{3} \mathrm{~S}: \quad \mathrm{C}, 46.28 ; \mathrm{H}$, 4.44. Found:. C, 46.33; H, 4.41.

$N(\pi)$-Methyl- $N(\alpha)$-(2,4,6-triisopropylbenzenesulfonyl)-L-hisitidinol $\quad(1, \quad$ Ar=2,4,6-i$\left.\operatorname{Pr}_{3} \mathbf{C}_{6} \mathbf{H}_{2}, \mathbf{X}=\mathbf{O H}\right)$ : white solid $(838 \mathrm{mg}, 2.0 \mathrm{mmol}, 50 \%$ yield $) ;[\alpha]_{\mathrm{D}}{ }^{20}=20.4\left(c=1.0\right.$ in $\left.\mathrm{CHCl}_{3}\right)$; IR (KBr) 3486, 3114, 3053, 2958, 2928, 2869, 1601, 1461, 1316, 1294, 1146, 1113, 1059, 1041, 664, $561 \mathrm{~cm}^{-1}$; ${ }^{1} \mathrm{H}$ NMR $\left(300 \mathrm{MHz}, \mathrm{CDCl}_{3}\right) \delta 1.248(\mathrm{~d}, J=6.9 \mathrm{~Hz}, 12 \mathrm{H}), 1.255(\mathrm{~d}, J=6.9 \mathrm{~Hz}, 6 \mathrm{H})$, 2.75-3.02 (m, 3H), 3.42-3.52 (m, 3H), $3.53(\mathrm{~s}, 3 \mathrm{H}), 4.13$ (septet, $J=6.9 \mathrm{~Hz}, 2 \mathrm{H}), 5.72(\mathrm{~d}, J=6.6$ $\mathrm{Hz}, 1 \mathrm{H}), 6.58(\mathrm{~s}, 1 \mathrm{H}), 7.16(\mathrm{~s}, 2 \mathrm{H}), 7.27(\mathrm{~s}, 1 \mathrm{H}) ;{ }^{13} \mathrm{C} \mathrm{NMR}\left(75 \mathrm{MHz}, \mathrm{CDCl}_{3}\right) \delta$ 23.6, 24.9, 25.0, $26.2,29.8,31.5,34.1,53.7,61.4,123.8,127.5,128.1,133.3,137.7,150.1,152.8$; MS (FAB+) $[\mathrm{M}+\mathrm{H}]^{+} \mathrm{m} / z$ 422. Anal. Calcd for $\mathrm{C}_{22} \mathrm{H}_{35} \mathrm{~N}_{3} \mathrm{O}_{3} \mathrm{~S}$ : C, 62.67; H, 8.37; N. Found: C, 62.61; H, 8.39 .

Procedure for the Preparation of $N(\pi)$-Methyl- $N(\alpha)$-benzoyl-L-hisitidinol (5, Ar=Ph, $\mathbf{X}=\mathbf{O H})$. To a solution of $\mathbf{3}(621 \mathrm{mg}, 4 \mathrm{mmol})$ in pyridine $(20 \mathrm{~mL})$ was added benzoyl chloride $(0.638 \mathrm{~mL}, 5.5 \mathrm{mmol})$ at $0{ }^{\circ} \mathrm{C}$. After the mixture was stirred for $5 \mathrm{~h}$ at room temperature, the solvent was removed under reduced pressure. The crude product was dissolved in EtOAc, and washed with water and brine. The organic phase was dried over $\mathrm{Na}_{2} \mathrm{SO}_{4}$, filtrated, and 
concentrated under reduced pressure. The reside was purified by flash column chromatography on $\mathrm{NH}$ silica gel (eluent: EtOAc) to give $\mathbf{5}$ in good yield. TLC (silica gel $\mathrm{NH}_{2} \mathrm{~F}_{2545}$, EtOAc-MeOH=10:1) $R_{\mathrm{f}}=0.21 ;[\alpha]_{\mathrm{D}}{ }^{20}=-28.2\left(c=1.0\right.$ in $\mathrm{CHCl}_{3}$ ); IR (neat) 3500-3300 (br), 2923, 2852, 1638, $1542 \mathrm{~cm}^{-1} ;{ }^{1} \mathrm{H}$ NMR $\left(300 \mathrm{MHz}, \mathrm{CDCl}_{3}\right) \delta 2.99(\mathrm{~s}, 1 \mathrm{H}), 3.01(\mathrm{~d}, J=3.0 \mathrm{~Hz}, 1 \mathrm{H}), 3.72$ (s, $3 \mathrm{H}), 3.77(\mathrm{~d}, J=1.5 \mathrm{~Hz}, 1 \mathrm{H}), 3.78(\mathrm{~d}, J=1.2 \mathrm{~Hz}, 1 \mathrm{H}), 4.23(\mathrm{~m}, 1 \mathrm{H}), 6.77(\mathrm{~d}, J=7.8 \mathrm{~Hz}, 1 \mathrm{H}), 6.83$ (s, 1H), $7.43(\mathrm{~m}, 3 \mathrm{H}), 7.52(\mathrm{~m}, 1 \mathrm{H}), 7.74(\mathrm{~m}, 1 \mathrm{H}) ;{ }^{13} \mathrm{C} \mathrm{NMR}\left(\mathrm{CDCl}_{3}\right)$ 25.0, 31.6, 50.8, 61.5, 126.9, 127.5, 128.5 (2C), 128.7, 131.6, 134.1, 137.8, 167.7. Anal. Calcd for $\mathrm{C}_{14} \mathrm{H}_{17} \mathrm{~N}_{3} \mathrm{O}_{2}$ : C, 64.85; $\mathrm{H}$, 6.61. Found: C, 64.90; H, 6.59.

General Procedure for the Preparation of (S)-1-tert-Butyldiphenylsilyloxy-3-(3'-methyl3'H-imidazol-4'-yl)-2-(arenesulfonylamino)propane (1, $\left.\mathrm{X}=t-\mathrm{BuPh}_{2} \mathrm{Si}\right)$ : $\quad$ To a solution of $N(\pi)$ methyl- $N(\alpha)$-(arenesulfonyl)-L-hisitidinol $(\mathbf{1}, \mathrm{X}=\mathrm{OH})(0.95 \mathrm{mmol})$ in DMF $(5 \mathrm{~mL})$ was added tertbutylchlorodiphenylsilane $(304 \mu \mathrm{L}, 1.17 \mathrm{mmol})$ and imidazole $(163 \mathrm{mg}, 2.4 \mathrm{mmol})$ at $0{ }^{\circ} \mathrm{C}$. After the mixture was stirred for $6 \mathrm{~h}$ at room temperature, the solvent was removed under reduced pressure to give the crude product. The residue was purified by flash column chromatography on $\mathrm{NH}$ silica gel (eluent: hexane-EtOAc $=1: 1)$ to give $\mathbf{1}\left(\mathrm{X}=t-\mathrm{BuPh}_{2} \mathrm{Si}\right)$.

(S)-1-tert-Butyldiphenylsilyloxy-3-(3'-methyl-3' $H$-imidazol-4'-yl)-2-

(benzenesulfonylamino)propane (1a): $\quad \mathrm{TLC}$ ( silica gel $\mathrm{NH}_{2} \mathrm{~F}_{254 \mathrm{~S}}$, hexane:EtOAc=1:2) $R_{\mathrm{f}}=0.15$; Purification by column chromatography on Cromatorex ${ }^{\circledR}$ NH-DM1020 (hexane:EtOAc=1:2 1:4); $[\alpha]^{20}=2.34\left(c=0.51, \mathrm{CHCl}_{3}\right)$; IR $(\mathrm{KBr}) 3069,2930,2857,1509,1428,1324,1158,1113,1070,823$, 706, $588 \mathrm{~cm}^{-1}$; ${ }^{1} \mathrm{H}$ NMR (300 MHz, $\mathrm{CDCl}_{3}$ ) $\delta 1.04$ (s, 9H), 2.84 (dd, J=5.4, $\left.15.0 \mathrm{~Hz}, 1 \mathrm{H}\right), 2.96$ (dd, $J=7.8,15.0 \mathrm{~Hz}, 1 \mathrm{H}), 3.28-3.37$ (m, 1H), 3.41 (s, 3H), 3.44 (dd, $J=4.8,10.5 \mathrm{~Hz}, 1 \mathrm{H}), 3.59$ (dd, $J=3.9$, $10.5 \mathrm{~Hz}, 1 \mathrm{H}), 5.42$ (br, 1H), 6.54 (s, 1H), 7.25 (s, 1H), 7.34-7.58 (m, 13H), 7.66 (d, J=8.1 Hz, 2H). ${ }^{13} \mathrm{C} \mathrm{NMR}\left(75 \mathrm{MHz}, \mathrm{CDCl}_{3}\right.$ ) 19.2, 26.4, 26.9 (3C), 31.3, 53.8, 63.7, 126.7 (2C), 127.0, 127.9 (4C), 128.0, 129.0 (2C), 130.0 (2C), 132.5 (2C), 135.4 (4C), 138.0 (2C), 140.1. Anal. Calcd for $\mathrm{C}_{29} \mathrm{H}_{35} \mathrm{~N}_{3} \mathrm{O}_{3} \mathrm{SSi}$ : C, 65.26; H, 6.61. Found: C, 65.18; H, 6.66.

\section{(S)-(+)-1-tert-Butyldiphenylsilyloxy-3-(3'-methyl-3'H-imidazol-4'-yl)-2-(4'’-}

trifluoromethylbenzenesulfonylamino)propane (1b): $\quad \mathrm{TLC}$ (silica gel $\mathrm{NH}_{2} \mathrm{~F}_{254 \mathrm{~S}}$, hexane:EtOAc=1:2) $R_{\mathrm{f}}=0.26$; Purification by column chromatography on silica gel Cromatorex ${ }^{\circledR}$ NH-DM1020 (hexane:EtOAc=1:2 1:4) and recrystallization $\left(\mathrm{CHCl}_{3}-\right.$ hexane); $[\alpha]^{20}{ }_{\mathrm{D}}=1.72(c 0.93$, $\left.\mathrm{CHCl}_{3}\right) ;{ }^{1} \mathrm{H} \mathrm{NMR}\left(300 \mathrm{~Hz}, \mathrm{CDCl}_{3}\right) \delta 1.02(\mathrm{~s}, 9 \mathrm{H}), 2.88(\mathrm{dd}, J=6.0,15.3 \mathrm{~Hz}, 1 \mathrm{H}), 2.98(\mathrm{dd}, J=6.6$, $15.3 \mathrm{~Hz}, 1 \mathrm{H}), 3.32-3.42(\mathrm{~m}, 1 \mathrm{H}), 3.44(\mathrm{~s}, 3 \mathrm{H}), 3.47(\mathrm{dd}, J=6.0,10.3 \mathrm{~Hz}, 1 \mathrm{H}), 3.57$ (dd, $J=4.2$, $10.3 \mathrm{~Hz}, 1 \mathrm{H}), 5.55$ (br, 1H), 6.57 (s, 1H), 7.21 (s, 1H), 7.33-7.39 (m, 4H), 7.41-7.48 (m, 2H), 7.50-7.56 (m, 4H), $7.57(\mathrm{~d}, J=8.2 \mathrm{~Hz}, 2 \mathrm{H}), 7.73(\mathrm{~d}, J=8.2 \mathrm{~Hz}, 2 \mathrm{H}) ;{ }^{13} \mathrm{C} \mathrm{NMR}\left(75 \mathrm{MHz}, \mathrm{CDCl}_{3}\right) \delta$ 19.2, 26.2, 26.8 (3C), 31.3, 54.1, 63.9, 123.1 (q, $J=273 \mathrm{~Hz}), 126.1$ (q, J=3.7 Hz, 2C), 126.9, 127.1 (2C), 127.9 (4C), 128.1, 130.1 (2C), 132.46, 132.53, 133.9 (q, $J=33.0 \mathrm{~Hz}), 135.4$ (4C), 134.0, 
144.1. Anal. Calcd for $\mathrm{C}_{30} \mathrm{H}_{34} \mathrm{~F}_{3} \mathrm{~N}_{3} \mathrm{O}_{3} \mathrm{SSi}$ : C, 59.88; H, 5.69. Found: C, 59.83; H, 5.73.

(S)-1-tert-Butyldiphenylsilyloxy-3-(3'-methyl-3' H-imidazol-4'-yl)-2-(2',4',,6'-

triisopropylbenzenesulfonylamino)propane (1d): white solid (615 $\mathrm{mg}, 0.93 \mathrm{mmol}, 98 \%$ yield): $[\alpha]_{\mathrm{D}}{ }^{20}=4.8\left(c=1.0, \mathrm{CHCl}_{3}\right) ;{ }^{1} \mathrm{H} \mathrm{NMR}\left(300 \mathrm{MHz}, \mathrm{CDCl}_{3}\right) \delta 1.05(\mathrm{~s}, 9 \mathrm{H}), 1.20(\mathrm{~d}, J=6.9 \mathrm{~Hz}, 6 \mathrm{H})$, $1.23(\mathrm{~d}, J=6.9 \mathrm{~Hz}, 6 \mathrm{H}), 1.25(\mathrm{~d}, J=6.9 \mathrm{~Hz}, 6 \mathrm{H}), 2.80-3.02(\mathrm{~m}, 3 \mathrm{H}), 3.43(\mathrm{~s}, 3 \mathrm{H}), 3.61$ (brs $3 \mathrm{H})$, 4.10 (septet, $J=6.9 \mathrm{~Hz}, 2 \mathrm{H}), 4.95-5.15$ (br, $1 \mathrm{H}), 6.48$ (s, 1H), 7.08 (s, 2H), 7.26-7.44 (m, 8H), 7.52-7.68 (m, 5H); ${ }^{13} \mathrm{C} \mathrm{NMR}\left(75 \mathrm{MHz}, \mathrm{CDCl}_{3}\right) \delta$ 19.3, 23.6, 24.9, 26.2, 27.0, 29.8, 31.3, 34.2, 53.3, 63.6, 123.8, 127.3, 127.9, 128.0, 130.0, 130.1, 132.5, 132.6, 133.4, 135.47, 135.50, 138.0, 150.1, 152.9; IR (KBr) 4325, 3072, 3053, 2959, 2928, 2859, 2739, 1601, 1511, 1463, 1427, 1322, 1152, 1113, 1072, 741, 703, 661, $560506 \mathrm{~cm}^{-1}$; MS (FAB+) $[\mathrm{M}+\mathrm{H}]^{+} \mathrm{m} / z$ 660. Anal. Calcd for $\mathrm{C}_{38} \mathrm{H}_{53} \mathrm{~N}_{3} \mathrm{O}_{3} \mathrm{SSi}: \mathrm{C}, 69.15 ; \mathrm{H}, 8.09$. Found: C, 69.19; H, 8.03.

General Procedure for the Preparation of (S)-3-(3'-Methyl-3'H-imidazol-4'-yl)-2(arenesulfonylamino)propyl Isobutyrate (1, X=OCOi-Pr). To a solution of $N(\pi)$-methyl- $N(\alpha)$ (arenesulfonyl)-L-hisitidinol $(\mathbf{1}, \mathrm{X}=\mathrm{OH})(1 \mathrm{mmol})$ in $\mathrm{CHCl}_{3}(10 \mathrm{~mL})$ was added isobutyryl chloride $(105 \mu \mathrm{L}, 1 \mathrm{mmol})$ and $\mathrm{Et}_{3} \mathrm{~N}(101 \mu \mathrm{L}, 1 \mathrm{mmol})$ at $0{ }^{\circ} \mathrm{C}$. After the mixture was stirred for $6 \mathrm{~h}$ at room temperature, the solvent was removed under reduced pressure. The reside was purified by flah column chromatography on $\mathrm{NH}$ silica gel (eluents: EtOAc-MeOH) to give $\mathbf{1}(\mathrm{X}=\mathrm{OCO} i$ - $\mathrm{Pr}$ ).

(S)-3-(3'-Methyl-3'H-imidazol-4'-yl)-2-(2',4',,6'-

triisopropylbenzenesulfonylamino)propyl Isobutyrate (1c): white solid (143 $\mathrm{mg}, 0.29 \mathrm{mmol}$, $29 \%$ yield); TLC (silica gel $\mathrm{NH}_{2} \mathrm{~F}_{254 \mathrm{~S}}$, hexane:EtOAc=1:2) $R_{\mathrm{f}}=0.22 ;[\alpha]_{\mathrm{D}}{ }^{20}=15.2(c=1.0$ in $\left.\mathrm{CHCl}_{3}\right) ;{ }^{1} \mathrm{H} \mathrm{NMR}\left(300 \mathrm{MHz}, \mathrm{CDCl}_{3}\right) \delta 7.33(\mathrm{~s}, 1 \mathrm{H}), 7.15(\mathrm{~s}, 1 \mathrm{H}), 6.70(\mathrm{~d}, J=8.1 \mathrm{~Hz}, 1 \mathrm{H}), 6.45(\mathrm{~s}$, $1 \mathrm{H}), 4.17(\mathrm{~m}, 2 \mathrm{H}), 4.05(\mathrm{dd}, J=4.7,11.3 \mathrm{~Hz}, 1 \mathrm{H}), 3.95(\mathrm{dd}, J=6.5,11.3 \mathrm{~Hz}, 1 \mathrm{H}), 5.79(\mathrm{~m}, 1 \mathrm{H})$, $3.65(\mathrm{~s}, 3 \mathrm{H}), 3.01(\mathrm{dd}, J=8.0,15.5 \mathrm{~Hz}, 1 \mathrm{H}), 2.90(\mathrm{~m}, 2 \mathrm{H}), 2.15(\mathrm{~m}, 1 \mathrm{H}), 1.24(\mathrm{~m}, 18 \mathrm{H}), 1.04(\mathrm{~d}, J$ $=7.2 \mathrm{~Hz}, 3 \mathrm{H}), 0.99(\mathrm{~d}, J=7.2 \mathrm{~Hz}, 3 \mathrm{H}) ;{ }^{13} \mathrm{C} \mathrm{NMR}\left(75 \mathrm{MHz}, \mathrm{CDCl}_{3}\right) \delta$ 18.76, 18.83, 23.6, 24.8, 25.0, 27.2, 29.7, 31.7, 33.5, 34.1, 51.5, 64.2, 123.8, 126.6, 128.0, 134.0, 137.9, 149.8, 152.6, 176.7; IR (KBr) 3436, 2961, 2929, 2871, 1735, 1601, 1466, 1321, 1194, 1151, 1113, 663, $570 \mathrm{~cm}^{-1}$; MS $(\mathrm{FAB}+)[\mathrm{M}+\mathrm{H}]^{+} \mathrm{m} / \mathrm{z}$ 492. Anal. Calcd for $\mathrm{C}_{26} \mathrm{H}_{41} \mathrm{~N}_{3} \mathrm{O}_{4} \mathrm{~S}: \quad \mathrm{C}, 63.51 ; \mathrm{H}, 8.40$. Found: 63.55; H, 8.34 .

(S)-2-[N-Isobutyryl(2',4',6'-triisopropylbenzenesulfonyl)amino]-3-(3'--methyl-3' $H$ -

imidazol-4"'-yl)propyl Isobutyrate (4): white solid (185 mg, $0.33 \mathrm{mmol}, 33 \%$ yield); TLC (silica gel $\mathrm{NH}_{2} \mathrm{~F}_{254 \mathrm{~S}}$, hexane:EtOAc=1:2) $R_{\mathrm{f}}=0.37 ;[\alpha]_{\mathrm{D}}{ }^{20}=-3.9\left(c=1.0\right.$ in $\left.\mathrm{CHCl}_{3}\right) ;{ }^{1} \mathrm{H}$ NMR $(300 \mathrm{MHz}$, $\left.\mathrm{CDCl}_{3}\right) \delta 7.32(\mathrm{~s} 1 \mathrm{H}), 7.23(\mathrm{~s}, 2 \mathrm{H}), 6.59(\mathrm{~s}, 1 \mathrm{H}), 4.32(\mathrm{~m}, 3 \mathrm{H}), 3.95(\mathrm{~m} \mathrm{2H}), 3.48(\mathrm{~s}, 3 \mathrm{H}), 3.44(\mathrm{~m}$, 2H), $2.92(\mathrm{~m}, 1 \mathrm{H}), 2.71(\mathrm{dd}, J=3.6,15.6 \mathrm{~Hz}, 1 \mathrm{H}), 2.38(\mathrm{~m}, 1 \mathrm{H}), 1.26(\mathrm{~m}, 18 \mathrm{H}), 1.12(\mathrm{~d}, J=6.6 \mathrm{~Hz}$, $3 \mathrm{H}), 1.06(\mathrm{~d}, J=1.5 \mathrm{~Hz}, 3 \mathrm{H}), 1.04(\mathrm{dd}, J=1.5,6.9 \mathrm{~Hz}, 6 \mathrm{H}) ;{ }^{13} \mathrm{C} \mathrm{NMR}\left(75 \mathrm{MHz}, \mathrm{CDCl}_{3}\right) \delta 18.6$, 18.8, 19.1, 19.6, 23.5, 24.6, 24.7, 25.9, 29.4, 31.2, 33.7, 34.2, 35.7, 57.7, 64.1, 124.4, 127.7, 128.1, 
131.6, 137.9, 151.1, 154.9, 176.3, 179.3; IR (KBr) 3439, 2964, 2934, 2873, 1742, 1689, 1601, 1466, 1386, 1367, 1336, 1204, 1145 952, 664, 588, $564 \mathrm{~cm}^{-1}$; MS (FAB+) $[\mathrm{M}+\mathrm{H}]^{+} \mathrm{m} / z$ 562. Anal. Calcd for $\mathrm{C}_{30} \mathrm{H}_{47} \mathrm{~N}_{3} \mathrm{O}_{5} \mathrm{~S}$ : C, 64.14; H, 8.43. Found; C, 64.23; H, 8.51.

\section{(S)-(+)-1-tert-Butyldiphenylsilyloxy-3-(3'-methyl-3' H-imidazol-4'-yl)-2-}

benzoylaminopropane (5): TLC (silica gel $\mathrm{NH}_{2} \mathrm{~F}_{254 \mathrm{~S}}$, hexane:EtOAc=1:2) $R_{\mathrm{f}}=0.17$; Purification by column chromatography on silica gel Cromatorex ${ }^{\circledR}$ NH-DM1020 (hexane:EtOAc=1:2 1:4); $[\alpha]^{20}{ }_{\mathrm{D}}=15.5\left(\mathrm{c} 0.62, \mathrm{CHCl}_{3}\right) ;{ }^{1} \mathrm{H} \mathrm{NMR}\left(300 \mathrm{MHz} \mathrm{CDCl}_{3}\right) \delta 1.13(\mathrm{~s}, 9 \mathrm{H}), 2.93(\mathrm{dd}, J=5.0,15.0 \mathrm{~Hz}$, $1 \mathrm{H}), 3.05(\mathrm{dd}, J=9.0,15.0 \mathrm{~Hz}, 1 \mathrm{H}), 3.70(\mathrm{~s}, 3 \mathrm{H}), 3.77(\mathrm{dd}, J=3.6,10.4 \mathrm{~Hz}, 1 \mathrm{H}), 3.86(\mathrm{dd}, J=2.7$, $10.4 \mathrm{~Hz}, 1 \mathrm{H}), 4.20(\mathrm{~m}, 1 \mathrm{H}), 6.64(\mathrm{br}, 1 \mathrm{H}), 6.66(\mathrm{~s}, 1 \mathrm{H}), 7.42(\mathrm{~m}, 10 \mathrm{H}), 7.64(\mathrm{~m}, 6 \mathrm{H}) ;{ }^{13} \mathrm{C} \mathrm{NMR}(75$ $\left.\mathrm{MHz}, \mathrm{CDCl}_{3}\right) \delta 19.3,25.5,26.9$ (3C), 31.4, 50.0, 63.2, 126.7 (2C), 127.87, 127.94 (4C), 128.1, 128.6 (2C), 130.0, 130.1, 131.6, 132.6, 132.9, 134.1, 135.46 (2C), 135.53 (2C), 138.1, 166.9. Anal. Calcd for $\mathrm{C}_{30} \mathrm{H}_{35} \mathrm{~N}_{3} \mathrm{O}_{2} \mathrm{Si}$ : C, 72.40; H, 7.09. Found: C, 72.48; H, 7.06.

General Procedure for the Preparation of 1-( $N$-Pyrrolidine-1'-carbonyloxy)-2-alcohols (6c, 8, 10, 12, 14) Derived from meso-1,2-Diols: The treatment of meso-1,2-diols (20 mmol) with bis(trichloromethyl)carbonate (triphosgene) $(20 \mathrm{mmol})$ in dichloromethane $(100 \mathrm{~mL})$ in the presence of pyridine $(10 \mathrm{~mL})$ at room temperature were converted to the corresponding cyclic carbonates in quantitative yield. ${ }^{2}$ Subsequent aminolysis of cyclic carbonates $(20 \mathrm{mmol})$ with pyrrolidine $(10 \mathrm{~mL})$ in THF $(40 \mathrm{~mL})$ under reflux conditions afforded 1-(N-pyrrolidine-1'carbonyloxy)-2-alcohols $(\mathbf{6 c}, \mathbf{8}, \mathbf{1 0}, \mathbf{1 2}, \mathbf{1 4})$ in quantitative yield.

\section{General Procedure for the Kinetic Resolution of Racemic Alcohols with Isobutyric} Anhydride Induced by Nucleophilic Catalysts. To a solution of racemic alcohol (0.25 mmol) and catalyst $(0.0125 \mathrm{mmol})$ in toluene $(2.5 \mathrm{~mL})$ was added $i$ - $\operatorname{Pr}_{2} \mathrm{NEt}(21.8 \mu \mathrm{L}, 0.125 \mathrm{mmol})$ and isobutyric anhydride $(20.7 \mu \mathrm{L}, 0.125 \mathrm{~mL})$. After being stirred for $3 \mathrm{~h}$ at room temperature or $0{ }^{\circ} \mathrm{C}$ (for each reaction temperature, see Tables 2-4), the reaction mixture was treated with $0.1 \mathrm{M} \mathrm{HCl}$ aqueous solution and extracted with EtOAc. The organic layer was washed with saturated aqueous $\mathrm{NaHCO}_{3}$, dried over $\mathrm{NaSO}_{4}$, and concentrated under reduced pressure. Ee values of the recovered alcohol and the acylated product were determined by HPLC analysis of crude products. The conversion $(c)$ was estimated by the following equation, $c(\%)=[$ ee (recovered alcohol)]/[ee (recovered alcohol) + ee (acylated product)]. ${ }^{3}$ The $S\left(k_{\text {fast }} / k_{\text {slow }}\right)$ value was estimated by the following equation, $S=\ln \left[(1-c)\left(1-\mathrm{ee}_{\text {alcohol }}\right)\right] / \ln \left[(1-c)\left(1+\mathrm{ee}_{\text {alcohol }}\right)\right]^{3}$

cis-1-[p-(Dimethylamino)benzoyloxy]-2-cyclohexanol (6a): ${ }^{4} \quad$ TLC (hexane-EtOAc=2:1) $R_{\mathrm{f}}=0.11 ;{ }^{1} \mathrm{H}$ NMR $\left(300 \mathrm{MHz}, \mathrm{CDCl}_{3}\right) \delta 1.34-1.52(\mathrm{~m}, 2 \mathrm{H}), 1.60-1.78(\mathrm{~m}, 4 \mathrm{H}), 1.84(\mathrm{q}, J=8.6 \mathrm{~Hz}$, $1 \mathrm{H}), 1.99$ (q, $J=9.8 \mathrm{~Hz}, 1 \mathrm{H}), 2.17$ (d, $J=4.2 \mathrm{~Hz}, 1 \mathrm{H}), 3.05(\mathrm{~s}, 6 \mathrm{H}), 3.91-3.98(\mathrm{~m}, 1 \mathrm{H}), 5.15-5.19$ (m, 1H), $6.52(\mathrm{~d}, J=9.1 \mathrm{~Hz}, 2 \mathrm{H}), 7.93(\mathrm{~d}, J=9.1 \mathrm{~Hz}, 2 \mathrm{H})$; HPLC (Daicel Chiralpak OD-H, 
hexane:2-propanol $=20: 1$, flow rate $1.0 \mathrm{~mL} / \mathrm{min}) t_{\mathrm{R}}=26.9((1 R, 2 S)$, minor $)$ and $55.5((1 S, 2 R)$, major) min.

cis-1-[p-(Dimethylamino)benzoyloxy]-2-cyclohexyl Isobutyrate $\quad(7 a):^{4} \quad$ TLC (hexane-EtOAc=2:1) $R_{\mathrm{f}}=0.60 ;[\alpha]_{\mathrm{D}}^{20}=-48.0\left(c\right.$ 1.0, $\left.\mathrm{CHCl}_{3}\right)$ for 7a of $84 \%$ ee; HPLC (Daicel Chiralcel OD-H, hexane:2-propanol = 20:1, flow rate $1.0 \mathrm{~mL} / \mathrm{min}) t_{\mathrm{R}}=9.4((1 R, 2 S)$, minor $)$ and 12.1 ((1S,2R), major) min; IR (film) 3019, 2943, 1725, 1697, 1608, 1526, 1368, 1281, 1216, 1184, 1109, 760, $668 \mathrm{~cm}^{-1}$; ${ }^{1} \mathrm{H}$ NMR $\left(300 \mathrm{MHz}, \mathrm{CDCl}_{3}\right) \delta 1.15$ (q, J=3.6 Hz, 6H), 1.42-15.6 (m, 2H), 1.63-1.81 (m, 4H), 1.87-2.02 (m, 2H), 2.54 (septet, J=6.9 Hz, 1H), $3.04(\mathrm{~s}, 6 \mathrm{H}), 5.07-5.13(\mathrm{~m}, 1 \mathrm{H})$, 5.19-5.26 (m, 1H), $6.64(\mathrm{~d}, J=6.9 \mathrm{~Hz}, 2 \mathrm{H}), 7.90(\mathrm{~d}, J=8.7 \mathrm{~Hz}, 2 \mathrm{H}) ;{ }^{13} \mathrm{C} \mathrm{NMR}\left(75 \mathrm{MHz}, \mathrm{CDCl}_{3}\right) \delta$ 18.9, 19.0, 27.79 (2C), 27.83, 27.9, 34.2, 40.0 (2C), 70.7, 71.0, 110.6 (2C), 117.2, 131.2 (2C), 153.2, $160.0,176.3$.

cis-1-Dimethylcarbamoyloxy-2-cyclohexanol (6b): $\quad$ TLC (hexane-EtOAc $=2: 1) R_{\mathrm{f}}=0.11$; GC (CHIRALDEX $\gamma$-TA $(20 \mathrm{~m})$, inj. temp. $140{ }^{\circ} \mathrm{C}$, col. temp. $\left.110{ }^{\circ} \mathrm{C}, \mathrm{N}_{2}(80 \mathrm{~Pa})\right) t_{\mathrm{R}}=29.4((1 S, 2 R)-6 \mathbf{b}$, minor), $31.6\left((1 R, 2 S)-6 \mathbf{b}\right.$, major) min; ${ }^{1} \mathrm{H}$ NMR (300 MHz, $\left.\mathrm{CDCl}_{3}\right) \delta 1.25-1.50(\mathrm{~m}, 2 \mathrm{H}), 1.50-1.64$ (m, 2H), 1.64-1.80 (m, 3H), 1.80-1.90 (m, 1H), 2.66 (s, 1H), 2.94 (s, 3H), 2.95 (s, 3H), 3.83 (br, 1H), 4.89-4.95 (m, 1H); $\left.{ }^{13} \mathrm{C} \mathrm{NMR} \mathrm{(75} \mathrm{MHz,} \mathrm{CDCl}_{3}\right) \delta 21.4,22.0,28.1,29.9,35.9,36.4,70.2,74.6$, 156.8. Anal. Calcd for $\mathrm{C}_{9} \mathrm{H}_{17} \mathrm{NO}_{3}$ : C, 57.73; H, 9.15. Found: C, 57.78; H, 9.22.

cis-1-Dimethylcarbamoyloxy-2-cyclohexyl Isobutyrate (7b): HPLC (Daicel Chiralpack AD-H, hexane:2-propanol = 40:1, flow rate $0.25 \mathrm{~mL} / \mathrm{min}) t_{\mathrm{R}}=57.8((1 S, 2 R)-7 \mathbf{b}$, major $), 61.0((1 R, 2 S)-7 \mathbf{b}$, minor) min. Anal. Calcd for $\mathrm{C}_{13} \mathrm{H}_{23} \mathrm{NO}_{4}$ : C, 60.68; H, 9.01. Found: C, 60.59; H, 9.14.

cis-N-(2-Hydroxycyclohexanoxycarbonyl)pyrrolidine (6c): TLC (hexane-EtOAc = 2:1) $R_{\mathrm{f}}=0.17 ;[\alpha]_{\mathrm{D}}^{20}=-2.7\left(c\right.$ 1.0, $\mathrm{CHCl}_{3}$ ) for 97\% ee; HPLC (Daicel Chiralpack AS-H, hexane:2propanol $=20: 1$, flow rate $=1.0 \mathrm{~mL} / \mathrm{min}) t_{\mathrm{R}}=24.7((1 S, 2 R)-\mathbf{6 c}$, minor $), 30.1((1 R, 2 S)-\mathbf{6 c}$, major $)$ min; IR (film) 3500-3350 (br), 2938, 2871, 1680, 1429, 1360, 1181, 1129, 1109, 984, $768 \mathrm{~cm}^{-1} ;{ }^{1} \mathrm{H}$ NMR (300 MHz, $\left.\mathrm{CDCl}_{3}\right) \delta$ 1.24-2.15 (m, 12H), $2.78(\mathrm{~s}, 1 \mathrm{H}), 3.40(\mathrm{t}, J=6.6 \mathrm{~Hz}, 4 \mathrm{H}), 3.83(\mathrm{br}, 1 \mathrm{H})$, $4.92(\mathrm{dt}, J=2.4,6.6 \mathrm{~Hz}, 1 \mathrm{H}) ;{ }^{13} \mathrm{C} \mathrm{NMR}\left(75 \mathrm{MHz}, \mathrm{CDCl}_{3}\right) \delta$ 21.3, 22.0, 24.9, 25.6, 28.2, 29.8, 45.8, 46.1, 70.1, 74.1, 155.1. Anal. Calcd for $\mathrm{C}_{11} \mathrm{H}_{19} \mathrm{NO}_{3}$ : C, 61.95; H, 8.98. Found: C, 61.91; H, 9.01 .

cis- $N$-(2-Isobutyryloxycyclohexanoxycarbonyl)pyrrolidine (7c): $\quad$ TLC (hexane-EtOAc $=2: 1$ ) $R_{\mathrm{f}}=0.27 ;[\alpha]_{\mathrm{D}}^{20}=-19.5\left(c 1.0, \mathrm{CHCl}_{3}\right)$ for $72 \%$ ee; HPLC (Daicel Chiralpack AS-H, hexane:2propanol $=20: 1$, flow rate $=0.5 \mathrm{~mL} / \mathrm{min}) t_{\mathrm{R}}=13.5((1 R, 2 S)-7 \mathbf{c}$, minor $), 14.5((1 S, 2 R)-7 \mathbf{c}$, major $)$ min; IR $\left(\mathrm{CHCl}_{3}\right)$ 2876, 2943, 2875, 1728, 1694, 1425, 1372, 1196, 1128, 1105, $756 \mathrm{~cm}^{-1}$; ${ }^{1} \mathrm{H}$ NMR $\left(300 \mathrm{MHz}, \mathrm{CDCl}_{3}\right) \delta 1.16(\mathrm{~d}, J=3.3 \mathrm{~Hz}, 3 \mathrm{H}), 1.19$ (d, J=3.3 Hz, 3H), 1.36-1.52 (m, 2H), 1.52-1.75 (m, 4H), 1.75-1.94 (m, 6H), 2.56 (septet, $J=6.9 \mathrm{~Hz}, 1 \mathrm{H}), 3.31(\mathrm{t}, J=6.3 \mathrm{~Hz}, 2 \mathrm{H}), 3.38$ (t, $J=6.0 \mathrm{~Hz}$, 2H), 4.86-4.93 (m, 1H), $5.04-5.10(\mathrm{~m}, 1 \mathrm{H}) ;{ }^{13} \mathrm{C} \mathrm{NMR}\left(75 \mathrm{MHz}, \mathrm{CDCl}_{3}\right) \delta 18.9,19.0,21.2,22.2$, 24.9, 25.6, 27.8, 28.1, 34.2, 45.6, 46.0, 70.8, 71.7, 154.3, 176.1. Anal. Calcd for $\mathrm{C}_{15} \mathrm{H}_{25} \mathrm{NO}_{4}$ : C, 
63.58; H, 8.89. Found: C, 63.46; H, 8.97.

cis-1-(N-Pyrrolidine-1'-carbonyloxy)-2-cyclopentanol (8): ${ }^{4}$ TLC (hexane-EtOAc $\left.=2: 1\right)$ $R_{\mathrm{f}}=0.09 ;[\alpha]^{20}{ }_{\mathrm{D}}=-7.9\left(c 1.0, \mathrm{CHCl}_{3}\right)$ for $90 \%$ ee; HPLC (Daicel Chiralpak AD-H, hexane:2propanol $=20: 1$, flow rate $1.0 \mathrm{~mL} / \mathrm{min}, t_{\mathrm{R}}=16.8$ (major) and 24.8 (minor) $\min$; IR (KBr) 3450-3350, 2980, 2951, 2874, 1661, 1443, 1360, 1173, 1115, 1037, 860, 769, 606, $504 \mathrm{~cm}^{-1} ;{ }^{1} \mathrm{H}$ NMR (300 $\left.\mathrm{MHz}, \mathrm{CDCl}_{3}\right) \delta 1.49-1.61(\mathrm{~m}, 1 \mathrm{H}), 1.61-1.77(\mathrm{~m}, 1 \mathrm{H}), 1.77-2.02(\mathrm{~m}, 8 \mathrm{H}), 2.54(\mathrm{~d}, J=3.3 \mathrm{~Hz}, 1 \mathrm{H})$, 3.34-3.43 (m, 4H), 4.13-4.21 (m, 1H), $4.93(\mathrm{dt}, J=4.7,6.3 \mathrm{~Hz}, 1 \mathrm{H}) ;{ }^{13} \mathrm{C}$ NMR $\left(75 \mathrm{MHz}, \mathrm{CDCl}_{3}\right) \delta$ $19.4,24.9,25.7,28.5,30.5,45.8,46.2,73.7,77.4,155.1$.

cis-1-( $N$-Pyrrolidine-1'-carbonyloxy)-2-cyclopentyl Isobutyrate: ${ }^{4}$ TLC (hexane-EtOAc $=2: 1) R_{\mathrm{f}}=0.28 ;[\alpha]_{\mathrm{D}}^{20}=-32.4\left(c\right.$ 1.0, $\left.\mathrm{CHCl}_{3}\right)$ for $94 \%$ ee; HPLC (Daicel Chiralpak AS-H, hexane:2-propanol $=20: 1$, flow rate $1.0 \mathrm{~mL} / \mathrm{min}, t_{\mathrm{R}}=8.6$ (major) and 10.2 (minor) min; $\mathrm{IR}(\mathrm{KBr})$ 2973, 2876, 1736, 1708, 1419, 1345, 1198, 1155, 1128, 1109, $767 \mathrm{~cm}^{-1}$; ${ }^{1} \mathrm{H}$ NMR (300 MHz, $\left.\mathrm{CDCl}_{3}\right) \delta 1.15(\mathrm{~d}, J=1.8 \mathrm{~Hz}, 3 \mathrm{H}), 1.17(\mathrm{~d}, J=1.8 \mathrm{~Hz}, 3 \mathrm{H}), 1.56-1.72(\mathrm{~m}, 1 \mathrm{H}), 1.72-1.80(\mathrm{~m}, 1 \mathrm{H})$, 1.80-1.92 (m, 6H), 1.92-2.06 (m, 2H), 2.53 (septet, $J=6.9 \mathrm{~Hz}, 1 \mathrm{H}), 3.31(\mathrm{t}, J=6.3 \mathrm{~Hz}, 2 \mathrm{H}), 3.37$ $(\mathrm{t}, J=6.3 \mathrm{~Hz}, 2 \mathrm{H}), 5.08(\mathrm{dt}, J=4.2,6.0 \mathrm{~Hz}, 1 \mathrm{H}), 5.15(\mathrm{dt}, J=4.2,5.4 \mathrm{~Hz}, 1 \mathrm{H}) ;{ }^{13} \mathrm{C} \mathrm{NMR}(75 \mathrm{MHz}$, $\left.\mathrm{CDCl}_{3}\right) \delta 18.8,18.9,19.2,24.9,25.7,28.3,28.4,34.1,45.7,46.1,74.3,74.8,154.4,176.2$.

cis-1-( $N$-Pyrrolidine-1'-carbonyloxy)-2-cycloheptanol $(9):^{4} \quad$ TLC (hexane-EtOAc $\left.=2: 1\right)$, $R_{\mathrm{f}}=0.09 ;[\alpha]_{\mathrm{D}}^{20}=-8.8\left(c 1.0, \mathrm{CHCl}_{3}\right)$ for $93 \%$ ee; HPLC (two linear Daicel Chiralcel OD-H columns, hexane:2-propanol $=20: 1$, flow rate $1.0 \mathrm{~mL} / \mathrm{min}$ ) $t_{\mathrm{R}}=37.0$ (major) and 39.6 (minor) min; IR (film) 3500-3400 (br), 2933, 2871, 1678, 1429, 1180, 1129, 1106, $769 \mathrm{~cm}^{-1} ;{ }^{1} \mathrm{H} \mathrm{NMR} \mathrm{(300} \mathrm{MHz,}$ $\left.\mathrm{CDCl}_{3}\right) \delta 1.40-1.62(\mathrm{~m}, 4 \mathrm{H}), 1.62-1.84(\mathrm{~m}, 6 \mathrm{H}), 1.84-2.00(\mathrm{~m}, 4 \mathrm{H}), 3.09(\mathrm{~s}, 1 \mathrm{H}), 3.40(\mathrm{t}, J=6.6$ $\mathrm{Hz}, 4 \mathrm{H}), 3.88-3.96(\mathrm{~m}, 1 \mathrm{H}), 4.97(\mathrm{dt}, J=2.4,7.2 \mathrm{~Hz}, 1 \mathrm{H}) ;{ }^{13} \mathrm{C} \mathrm{NMR}\left(75 \mathrm{MHz}, \mathrm{CDCl}_{3}\right) 22.5,22.8$, 24.9, 25.7, 26.9, 28.9, 31.4, 73.5, 78.5, 155.4.

cis-1-( $N$-Pyrrolidine-1'-carbonyloxy)-2-cycloheptyl Isobutyrate: ${ }^{4}$ TLC (hexane-EtOAc $=$ $2: 1), R_{\mathrm{f}}=0.36 ;[\alpha]_{\mathrm{D}}^{20}=-17.9\left(c 1.0, \mathrm{CHCl}_{3}\right)$ for $92 \%$ ee; HPLC (two linear Daicel Chiralcel OD-H columns, hexane:2-propanol $=20: 1$, flow rate $1.0 \mathrm{~mL} / \mathrm{min}$ ) $t_{\mathrm{R}}=15.4$ (minor) and 16.3 (major) min; IR (film) 2936, 2872, 1733, 1703, 1419, 1195, 1157, 1100, $768 \mathrm{~cm}^{-1} ;{ }^{1} \mathrm{H} \mathrm{NMR}\left(300 \mathrm{MHz}, \mathrm{CDCl}_{3}\right) \delta$ $1.17(\mathrm{~d}, J=7.0 \mathrm{~Hz}, 3 \mathrm{H}), 1.18(\mathrm{~d}, J=7.0 \mathrm{~Hz}, 3 \mathrm{H}), 1.47-1.81(\mathrm{~m}, 8 \mathrm{H}), 1.81-2.01(\mathrm{~m}, 6 \mathrm{H}), 2.58$ (septet, $J=7.0 \mathrm{~Hz}, 1 \mathrm{H}), 3.26-3.43(\mathrm{~m}, 4 \mathrm{H}), 4.94-5.01(\mathrm{~m}, 1 \mathrm{H}), 5.10-5.16(\mathrm{~m}, 1 \mathrm{H}) ;{ }^{13} \mathrm{C}$ NMR $(75$ $\left.\mathrm{MHz}, \mathrm{CDCl}_{3}\right)$ 18.9, 19.0, 22.5, 22.7, 24.9, 25.7, 26.6, 28.8, 29.1, 34.2, 45.6, 46.0, 74.5, 75.2, 154.4, 176.2 .

(2RS,3SR)-2-(N-Pyrrolidine-1'-carbonyloxy)-3-butanol (10): ${ }^{5} \quad$ TLC (hexane-EtOAc=2:1) $R_{\mathrm{f}}=0.10 ;[\alpha]^{20}{ }_{\mathrm{D}}=-2.3\left(c 1.0, \mathrm{CHCl}_{3}\right)$ for $82 \%$ ee; HPLC (Daicel Chiralpak AD-H, hexane:2propanol $=20: 1$, flow rate $1.0 \mathrm{~mL} / \mathrm{min}, t_{\mathrm{R}}=15.9$ (major) and 20.8 (minor) min; IR (film) 3500-3350 (br), 2977, 2877, 1679, 1426, 1130, 1106, $769 \mathrm{~cm}^{-1} ;{ }^{1} \mathrm{H}$ NMR $\left(300 \mathrm{MHz}, \mathrm{CDCl}_{3}\right) \delta 1.16$ (d, $J=6.3$ $\mathrm{Hz}, 3 \mathrm{H}), 1.22(\mathrm{~d}, J=6.6 \mathrm{~Hz}, 3 \mathrm{H}), 1.82-1.95(\mathrm{~m}, 4 \mathrm{H}), 2.81(\mathrm{~s}, 1 \mathrm{H}), 3.33-3.43(\mathrm{~m}, 4 \mathrm{H}), 3.83-3.93$ 
$(\mathrm{m}, 1 \mathrm{H}), 4.84(\mathrm{dq}, J=2.7,12.9 \mathrm{~Hz}, 1 \mathrm{H}) ;{ }^{13} \mathrm{C} \mathrm{NMR}\left(75 \mathrm{MHz}, \mathrm{CDCl}_{3}\right) \delta 15.3,17.2,24.9,25.6,45.8$, 46.2, 70.0, 75.1, 155.2 .

(2RS,3SR)-2-(N-Pyrrolidine-1'-carbonyloxy)-3-butyl Isobutyrate: ${ }^{5}$ TLC (hexane-EtOAc $=2: 1) R_{\mathrm{f}}=0.33 ;[\alpha]_{\mathrm{D}}^{20}=-25.2\left(c\right.$ 1.0, $\left.\mathrm{CHCl}_{3}\right)$ for 93\% ee; HPLC (Daicel Chiralpak AD-H, hexane:2-propanol $=20: 1$, flow rate $1.0 \mathrm{~mL} / \mathrm{min}, t_{\mathrm{R}}=7.1$ (major) and 8.4 (minor) min; IR (film) 2978, 2877, 1734, 1705, 1416, 1196, 1160, 1103, $768 \mathrm{~cm}^{-1} ;{ }^{1} \mathrm{H}$ NMR $\left(300 \mathrm{MHz}, \mathrm{CDCl}_{3}\right) \delta 1.16(\mathrm{~d}, J$ $=7.2 \mathrm{~Hz}, 3 \mathrm{H}), 1.17(\mathrm{~d}, J=6.8 \mathrm{~Hz}, 3 \mathrm{H}), 1.21(\mathrm{~d}, J=6.3 \mathrm{~Hz}, 3 \mathrm{H}), 1.24(\mathrm{~d}, J=6.8 \mathrm{~Hz}, 3 \mathrm{H})$, 1.82-1.92 (m, 4H), 2.54 (septet, $J=7.0 \mathrm{~Hz}, 1 \mathrm{H}), 3.30(\mathrm{t}, J=6.3 \mathrm{~Hz}, 2 \mathrm{H}), 3.38(\mathrm{t}, J=6.3 \mathrm{~Hz}, 2 \mathrm{H})$, $4.88(\mathrm{dq}, J=4.1,6.5 \mathrm{~Hz}, 1 \mathrm{H}), 3.38(\mathrm{t}, J=6.3 \mathrm{~Hz}, 2 \mathrm{H}), 4.88(\mathrm{dq}, J=4.1,6.5 \mathrm{~Hz}, 1 \mathrm{H}), 5.03(\mathrm{dq}, J=$ 4.1, 65. Hz, 1H); ${ }^{13} \mathrm{C} \mathrm{NMR}\left(75 \mathrm{MHz}, \mathrm{CDCl}_{3}\right) \delta 15.3,15.5,18.8,19.0,24.9,25.6,34.1,45.6,46.0$, $71.3,72.0,154.3,176.3$.

$N$-(3-Hydroxy-3-phenylpropionyl)pyrrolidine (11): TLC (hexane-EtOAc $=2: 1) R_{\mathrm{f}}=0.22$; $[\alpha]_{\mathrm{D}}{ }^{20}=-51.5\left(c=1.0\right.$ in $\left.\mathrm{CHCl}_{3}\right)$ for $64 \%$ ee; HPLC (Daicel Chiralpak AD-H, hexane:2-propanol = 20:1, flow rate $1.0 \mathrm{~mL} / \mathrm{min}$ ) $t_{\mathrm{R}}=30.2$ (minor) and 32.1 (major) min; IR ( $\left.\mathrm{KBr}\right) 3300-3200(\mathrm{OH})$, $1609(\mathrm{C}=\mathrm{O}), 1474,1065,707 \mathrm{~cm}^{-1}$; ${ }^{1} \mathrm{H}$ NMR $\left(300 \mathrm{MHz}, \mathrm{CDCl}_{3}\right) \delta 1.80-2.00(\mathrm{~m}, 4 \mathrm{H}), 2.58(\mathrm{dd}, J=$ 8.7, 16.2 Hz, 1H), $2.65(\mathrm{dd}, J=3.6,16.2 \mathrm{~Hz}, 1 \mathrm{H}), 3.31(\mathrm{t}, J=6.6 \mathrm{~Hz}, 2 \mathrm{H}), 3.48(\mathrm{t}, J=6.3 \mathrm{~Hz}, 2 \mathrm{H})$, $4.97(\mathrm{~d}, J=3.0 \mathrm{~Hz}, 1 \mathrm{H}), 5.16(\mathrm{dt}, J=3.3,8.7 \mathrm{~Hz}, 1 \mathrm{H}), 7.24-7.45(\mathrm{~m}, 5 \mathrm{H}) ;{ }^{13} \mathrm{C} \mathrm{NMR}(75 \mathrm{MHz}$, $\left.\mathrm{CDCl}_{3}\right) \delta 24.3,25.8,43.0,45.5,46.5,70.3,125.6(2 \mathrm{C}), 127.4,128.4$ (2C), 143.1, 170.7. Anal. Calcd for $\mathrm{C}_{13} \mathrm{H}_{17} \mathrm{NO}_{2}$ : C, 71.21; H, 7.81. Found: C, 71.19; H, 7.99.

$N$-(3-Isobutyryloxy-3-phenylpropionyl)pyrrolidine: TLC (hexane-EtOAc $=1: 2) R_{\mathrm{f}}=0.35$; $[\alpha]_{\mathrm{D}}{ }^{20}=29.5\left(c=1.0\right.$ in $\left.\mathrm{CHCl}_{3}\right)$; HPLC (Daicel Chiralpak AD-H, hexane:2-propanol = 20:1, flow rate $1.0 \mathrm{~mL} / \mathrm{min}$ ) $t_{\mathrm{R}}=36.5$ (major) and 45.0 (minor) $\mathrm{min} ;{ }^{1} \mathrm{H} \mathrm{NMR}\left(300 \mathrm{MHz}, \mathrm{CDCl}_{3}\right) \delta 1.15(\mathrm{t}, J=$ $7.1 \mathrm{~Hz}, 6 \mathrm{H}), 1.76-1.95$ (m, 4H), 2.56 (septet, $J=7.1 \mathrm{~Hz}, 1 \mathrm{H}), 2.65$ (dd, $J=5.4,15.0 \mathrm{~Hz}, 1 \mathrm{H}), 2.94$ $(\mathrm{dd}, J=8.3,15.0 \mathrm{~Hz}, 1 \mathrm{H}), 3.22-3.31(\mathrm{~m}, 1 \mathrm{H}), 3.40-3.51(\mathrm{~m}, 3 \mathrm{H}), 6.21(\mathrm{dd}, J=5.4,8.3 \mathrm{~Hz}, 1 \mathrm{H})$, 7.24-7.41 (m, 5H); $\left.{ }^{13} \mathrm{C} \mathrm{NMR} \mathrm{(75} \mathrm{MHz,} \mathrm{CDCl}_{3}\right) \delta 18.8$ (2C), 24.3 , 26.0, 33.9, 42.0, 45.6, 46.7, 72.6, 126.2 (2C), 127.9, 128.5 (2C), 140.5, 167.6, 175.6. Anal. Calcd for $\mathrm{C}_{17} \mathrm{H}_{23} \mathrm{NO}_{3}$ : C, 70.56; $\mathrm{H}$, 8.01. Found: C, 70.67; H, 7.93 .

(2SR,3RS)- $N$-(3-Hydroxy-2-methyl-3-phenylpropionyl)pyrrolidine

(12):

TLC (hexane-EtOAc $=1: 2) \mathrm{R}_{\mathrm{f}}=0.35 ;[\alpha]_{\mathrm{D}}^{20}=-80.1\left(c=1.0\right.$ in $\left.\mathrm{CHCl}_{3}\right)$ for $80 \%$ ee; HPLC (Daicel Chiralpak AD-H, hexane:2-propanol = 20:1, flow rate $1.0 \mathrm{~mL} / \mathrm{min}$ ) $t_{\mathrm{R}}=26.6$ (major) and 28.4 (minor) min; IR (KBr) 3400-3300 (OH), 2976, 2872, 1613, 1469, 1447, 1047, 756, $702 \mathrm{~cm}^{-1} ;{ }^{1} \mathrm{H}$ NMR $\left(300 \mathrm{MHz}, \mathrm{CDCl}_{3}\right) \delta 1.25(\mathrm{~d}, J=7.2 \mathrm{~Hz}, 3 \mathrm{H}), 1.65-1.87(\mathrm{~m}, 4 \mathrm{H}), 2.86(\mathrm{dq}, J=7.2,2.1 \mathrm{~Hz}$, 1H), 2.96-3.05 (m, 1H), 3.23-3.32 (m, 1H), 3.34-3.41 (m, 2H), 4.64 (d, J=7.2 Hz, 1H), 4.77 (dd, $J$ $=5.1,6.9 \mathrm{~Hz}, 1 \mathrm{H}), 7.21-7.37(\mathrm{~m}, 5 \mathrm{H}) ;{ }^{13} \mathrm{C} \mathrm{NMR}\left(75 \mathrm{MHz}, \mathrm{CDCl}_{3}\right) \delta 15.3,24.1,25.8,44.8,45.4$, 46.5, 76.6, 125.9 (2C), 127.4, 128.2 (2C), 143.3, 174.1. Anal. Calcd for $\mathrm{C}_{14} \mathrm{H}_{19} \mathrm{NO}_{2}$ : C, 72.07; $\mathrm{H}$, 8.21. Found: $\mathrm{C}, 72.21 ; \mathrm{H}, 8.13$. 
$N$-(3-Isobutyryloxy-2-methyl-3-phenylpropionyl)pyrrolidine: TLC (hexane-EtOAc=1:1) $\mathrm{R}_{\mathrm{f}}=0.19 ;[\alpha]_{\mathrm{D}}^{20}=55.8\left(c=1.0\right.$ in $\left.\mathrm{CHCl}_{3}\right)$ for 82\% ee; HPLC (Daicel Chiralpak AD-H, hexane:2propanol $=20: 1$, flow rate $1.0 \mathrm{~mL} / \mathrm{min}$ ) $t_{\mathrm{R}}=27.4$ (minor) and 49.7 (major) min; IR $(\mathrm{KBr}) 2973$, $2875,1731,1628,1459,1438,1200,1162,703 \mathrm{~cm}^{-1} ;{ }^{1} \mathrm{H}$ NMR $\left(300 \mathrm{MHz}, \mathrm{CDCl}_{3}\right) \delta 0.91(\mathrm{~d}, J=7.2$ $\mathrm{Hz}, 3 \mathrm{H}), 1.07$ (d, $J=7.2 \mathrm{~Hz}, 3 \mathrm{H}), 1.09$ (d, $J=7.2 \mathrm{~Hz}, 3 \mathrm{H}), 1.82-1.94$ (m, 2H), 1.94-2.05 (m, 2H), 2.45 (septet, $J=6.9 \mathrm{~Hz}, 1 \mathrm{H}), 3.60$ (dq, $J=3.9,6.9 \mathrm{~Hz}, 1 \mathrm{H}), 3.45-3.56$ (m, 3H), 3.68-3.78 (m, 1H), $5.77(\mathrm{~d}, J=10.2 \mathrm{~Hz}, 1 \mathrm{H}), 7.26-7.39(\mathrm{~m}, 5 \mathrm{H}) ;{ }^{13} \mathrm{C} \mathrm{NMR}\left(75 \mathrm{MHz}, \mathrm{CDCl}_{3}\right) \delta$ 13.9, 18.5, 18.8, 24.4, 26.1, 33.9, 43.6, 45.8, 46.7, 78.4, 127.3 (2C), 128.1, 128.4 (2C), 138.9, 172.1, 174.9. Anal. Calcd for $\mathrm{C}_{18} \mathrm{H}_{25} \mathrm{NO}_{3}$ : C, 71.26; $\mathrm{H}, 8.31$. Found: C, 71.18; H, 8.43.

cis- $N$-(2'-Hydroxyindan-1'-yl)pyrrolidine-1-carboxamide (13): ${ }^{6} \quad$ TLC (EtOAc) $R_{\mathrm{f}}=0.40$; $[\alpha]^{20}{ }_{\mathrm{D}}=-36.6\left(c\right.$ 1.0, $\left.\mathrm{CHCl}_{3}\right)$ for $67 \%$ ee; HPLC (Daicel Chiralpack AD-H, hexane:2-propanol = 9:1, flow rate $=1.0 \mathrm{~mL} / \mathrm{min}) t_{\mathrm{R}}=11.9$ (major), 15.0 (minor) $\mathrm{min}$; IR $(\mathrm{KBr}) 3405,3205,1618,1523$, 1474, 1404, 1180, 1060, $744 \mathrm{~cm}^{-1}$; ${ }^{1} \mathrm{H}$ NMR (300 MHz, $\left.\mathrm{CDCl}_{3}\right) \delta$ 1.90-1.97 (m, 4H), 1.93 (s, 1H), $2.96(\mathrm{dd}, J=3.6,16.5 \mathrm{~Hz}, 1 \mathrm{H}), 3.17(\mathrm{dd}, J=5.6,16.5 \mathrm{~Hz}, 1 \mathrm{H}), 3.34-3.44(\mathrm{~m}, 4 \mathrm{H}), 4.61-4.68(\mathrm{~m}$, $1 \mathrm{H}), 4.71(\mathrm{~d}, J=7.5 \mathrm{~Hz}, 1 \mathrm{H}), 5.29(\mathrm{dd}, J=5.3,7.4 \mathrm{~Hz}, 1 \mathrm{H}), 7.19-7.36(\mathrm{~m}, 4 \mathrm{H}),{ }^{13} \mathrm{C} \mathrm{NMR}(75 \mathrm{MHz}$, $\left.\mathrm{CDCl}_{3}\right) \delta 25.5$ (2C), 39.1, 45.7 (2C), 58.8, 73.9, 124.7, 125.3, 127.1, 128.3, 140.4, 141.3, 157.2.

cis- $N$-(2'-Isobutyryloxyindan-1'-yl)pyrrolidine-1-carboxamide: ${ }^{6}$ TLC (hexane-EtOAc $=$ 1:2) $R_{\mathrm{f}}=0.34 ;[\alpha]_{\mathrm{D}}^{20}=63.2\left(c 1.0, \mathrm{CHCl}_{3}\right)$ for $84 \%$ ee; HPLC (Daicel Chiralpack AD-H, hexane:2propanol $=9: 1$, flow rate $=1.0 \mathrm{~mL} / \mathrm{min}) t_{\mathrm{R}}=16.7$ (major), 24.6 (minor) $\min ; \mathrm{IR}(\mathrm{KBr}) 3550-3300$ (br), 1729, 1642, 1622, 1524, 1403, 1189, 1151, $1037 \mathrm{~cm}^{-1} ;{ }^{1} \mathrm{H}$ NMR $\left(300 \mathrm{MHz}, \mathrm{CDCl}_{3}\right) \delta 1.03$ (d, $J=6.0 \mathrm{~Hz}, 3 \mathrm{H}), 1.05(\mathrm{~d}, J=6.0 \mathrm{~Hz}, 3 \mathrm{H}), 1.82-1.90(\mathrm{~m}, 4 \mathrm{H}), 2.40$ (septet, $J=7.0 \mathrm{~Hz}, 1 \mathrm{H}), 2.89$ (d, $J=17.4 \mathrm{~Hz}, 1 \mathrm{H}), 3.16(\mathrm{dd}, J=5.1,17.4 \mathrm{~Hz}, 1 \mathrm{H}), 3.23-3.38(\mathrm{~m}, 4 \mathrm{H}), 4.62(\mathrm{~d}, J=9.3 \mathrm{~Hz}, 1 \mathrm{H}), 5.48$ $(\mathrm{dt}, J=0.9,5.6 \mathrm{~Hz}, 1 \mathrm{H}), 5.58(\mathrm{dd}, J=5.6,9.2 \mathrm{~Hz}, 1 \mathrm{H}), 7.13-7.21(\mathrm{~m}, 3 \mathrm{H}), 7.25-7.30(\mathrm{~m}, 1 \mathrm{H}) ;{ }^{13} \mathrm{C}$ NMR (75 MHz, $\left.\mathrm{CDCl}_{3}\right) \delta$ 18.7, 19.1, 23.5 (2C), 34.0, 37.7, 45.6 (2C), 56.7, 76.0, 123.9, 124.9, $127.1,127.9,139.4,141.9,156.2,176.2$.

(2SR,3SR)-2-(N-Pyrrolidine-1'-carboxamino)-3-hydroxybutyric Acid Methyl Ester (14): TLC (hexane-EtOAc $=1: 5) R_{\mathrm{f}}=0.17 ;[\alpha]_{\mathrm{D}}^{20}=-32.0\left(c 1.0, \mathrm{CHCl}_{3}\right)$ for $51 \%$ ee; HPLC (Daicel Chiralpack AS-H, hexane:2-propanol $=5: 1$, flow rate $=0.5 \mathrm{~mL} / \mathrm{min}$ ) $t_{\mathrm{R}}=23.5$ (major), 27.7 (minor) $\min$; IR (KBr) 3400-3300 (br), 2987, 2956, 2879, 1751, 1616, 1526, 1433, 1191, $1163 \mathrm{~cm}^{-1} ;{ }^{1} \mathrm{H}$ NMR (300 MHz, CDCl $) \delta 1.11(\mathrm{~d}, J=6.3 \mathrm{~Hz}, 3 \mathrm{H}), 1.91-1.96(\mathrm{~m}, 4 \mathrm{H}), 3.36-3.42(\mathrm{~m}, 4 \mathrm{H}), 3.79$ (s, $3 \mathrm{H}), 4.17-4.27(\mathrm{~m}, 1 \mathrm{H}), 4.40(\mathrm{~d}, J=5.4 \mathrm{~Hz}, 1 \mathrm{H}), 4.68(\mathrm{dd}, J=3.3,6.0 \mathrm{~Hz}, 1 \mathrm{H}), 5.25(\mathrm{~d}, J=5.7 \mathrm{~Hz}$, $1 \mathrm{H}) ;{ }^{13} \mathrm{C} \mathrm{NMR}\left(75 \mathrm{MHz}, \mathrm{CDCl}_{3}\right) \delta 18.0,25.5$ (2C), 45.7 (2C), 52.6, 59.1, 69.1, 157.0, 171.6. Anal. Calcd for $\mathrm{C}_{10} \mathrm{H}_{18} \mathrm{~N}_{2} \mathrm{O}_{4}$ : C, 52.16; H, 7.88. Found: C, 52.11; H, 7.90.

(2SR,3SR)-2-( $N$-Pyrrolidine-1-carboxamino)-3-isobutyryloxybutyric Acid Methyl Ester: TLC (hexane-EtOAc $=1: 2) R_{\mathrm{f}}=0.34 ;[\alpha]_{\mathrm{D}}^{20}=1.1\left(c 1.0, \mathrm{CHCl}_{3}\right)$ for $80 \%$ ee; HPLC (Daicel 
Chiralpack AS-H, hexane:2-propanol $=5: 1$, flow rate $=0.5 \mathrm{~mL} / \mathrm{min}$ ) $t_{\mathrm{R}}=12.6$ (major), 15.6 (minor) min; IR (KBr) 3354, 3290, 2979, 2944, 2875, 1739, 1639, 1535, 1416, 1197, $1161 \mathrm{~cm}^{-1}$; ${ }^{1} \mathrm{H}$ NMR $\left(\mathrm{CDCl}_{3}, 300 \mathrm{MHz}\right) \delta 1.14(\mathrm{~d}, J=4.8 \mathrm{~Hz}, 3 \mathrm{H}), 1.32(\mathrm{~d}, J=4.8 \mathrm{~Hz}, 3 \mathrm{H}), 1.36(\mathrm{~d}, J=6.6 \mathrm{~Hz}, 3 \mathrm{H})$, 1.89-1.94 (m, 4H), 2.53 (septet, $J=7.0 \mathrm{~Hz}, 1 \mathrm{H}), 3.33-3.39$ (m, 4H), 3.77 (s, 3H), 4.69 (dd, J=3.6 , $8.1 \mathrm{~Hz}, 1 \mathrm{H}), 5.13(\mathrm{dq}, J=3.3,12.9 \mathrm{~Hz}, 1 \mathrm{H}), 5.21(\mathrm{~d}, J=8.1 \mathrm{~Hz}, 1 \mathrm{H}), 4.69$ (dd, $J=3.6,8.1 \mathrm{~Hz}, 1 \mathrm{H})$, $5.13(\mathrm{dq}, J=3.3,12.9 \mathrm{~Hz}, 1 \mathrm{H}), 5.21(\mathrm{~d}, J=8.1 \mathrm{~Hz}, 1 \mathrm{H}) ;{ }^{13} \mathrm{C} \mathrm{NMR}\left(75 \mathrm{MHz}, \mathrm{CDCl}_{3}\right) \delta 17.2,18.7$, 18.9, 25.5 (2C), 34.0, 45.5 (2C), 52.3, 57.3, 71.4, 155.7, 171.0, 176.9. Anal. Calcd for $\mathrm{C}_{14} \mathrm{H}_{24} \mathrm{~N}_{2} \mathrm{O}_{5}$ : C, 55.98; H, 8.05. Found: C, 55.91; H, 8.08.

3-Methyl-2-( $N$-pyrrolidine-1-carboxamino)-1-butanol (15): $\quad$ TLC $\left(\right.$ EtOAc) $R_{\mathrm{f}}=0.14 ;[\alpha]^{20}{ }_{\mathrm{D}}=$ -37.2 ( $c$ 1.0, $\mathrm{CHCl}_{3}$ ) for 88\% ee; HPLC (Daicel Chiralpack AS-H, hexane:2-propanol = 5:1, flow rate $=0.5 \mathrm{~mL} / \mathrm{min}$ ) $t_{\mathrm{R}}=11.1$ (major), 14.5 (minor) $\mathrm{min}$; IR $(\mathrm{KBr}) 3364,3292,2969,2869,1608$, 1525, 1408, 1335, $1086 \mathrm{~cm}^{-1} ;{ }^{1} \mathrm{H} \mathrm{NMR}\left(\mathrm{CDCl}_{3}, 300 \mathrm{MHz}\right) \delta 0.95(\mathrm{~d}, J=5.1 \mathrm{~Hz}, 3 \mathrm{H}), 0.97(\mathrm{~d}, J=$ $5.1 \mathrm{~Hz}, 3 \mathrm{H}), 1.84-1.99$ (m. 5H), 3.31-3.39 (m, 4H), 3.55-3.68 (m, 2H), 3.68-3.78 (m, 1H), 3.98 (t, $J=4.8 \mathrm{~Hz}, 1 \mathrm{H}), 4.39(\mathrm{~d}, J=6.0 \mathrm{~Hz}, 1 \mathrm{H}) ;{ }^{13} \mathrm{C} \mathrm{NMR}\left(75 \mathrm{MHz}, \mathrm{CDCl}_{3}\right) \delta 18.8,19.6,25.5$ (2C), 29.4, 45.6 (2C), 58.2, 65.4, 157.9. Anal. Calcd for $\mathrm{C}_{10} \mathrm{H}_{20} \mathrm{~N}_{2} \mathrm{O}_{2}$ : C, 59.97; H, 10.07. Found: C, $59.89 ; \mathrm{H}, 10.12$.

Isobutyryloxy-3-methyl-2-( $N$-pyrrolidine-1-carboxamino)butane: TLC (hexane-EtOAc $=1: 5)$ $R_{\mathrm{f}}=0.33 ;[\alpha]^{20}{ }_{\mathrm{D}}=30.6\left(c\right.$ 1.0, $\left.\mathrm{CHCl}_{3}\right)$ for $86 \%$ ee; HPLC (Daicel Chiralpack AS-H, hexane:2propanol $=5: 1$, flow rate $=0.5 \mathrm{~mL} / \mathrm{min}$ ) $t_{\mathrm{R}}=9.0$ (minor), 13.0 (major) min; IR $(\mathrm{KBr}) 3313,2969$, 2872, 1731, 1625, 1533, 1469, 1405, 1195, 1161, $1081 \mathrm{~cm}^{-1} ;{ }^{1} \mathrm{H}$ NMR $\left(\mathrm{CDCl}_{3}, 300 \mathrm{MHz}\right) \delta 0.96(\mathrm{~d}$, $J=6.6 \mathrm{~Hz}, 6 \mathrm{H}), 1.14(\mathrm{~d}, J=2.1 \mathrm{~Hz}, 3 \mathrm{H}), 1.17(\mathrm{~d}, J=2.1 \mathrm{~Hz}, 3 \mathrm{H}), 1.84$ (septet, $J=6.7 \mathrm{~Hz}, 1 \mathrm{H})$, 1.87-1.94 (m, 4H), 2.56 (septet, $J=7.0 \mathrm{~Hz}, 1 \mathrm{H}), 3.32$ (t, $J=6.6 \mathrm{~Hz}, 4 \mathrm{H}), 3.90-4.06(\mathrm{~m}, 2 \mathrm{H})$,

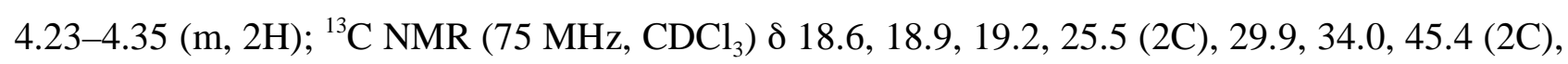
54.3, 64.7, 156.4, 177.5. Anal. Cald for $\mathrm{C}_{14} \mathrm{H}_{26} \mathrm{~N}_{2} \mathrm{O}_{3}$ : C, 62.19; H, 9.69. Found: C, 62.22; H, 9.75 .

\section{Procedure for the Preparation of Polystyrene-bound Catalyst 2. (4-} Methoxyphenyl)diisopropylsilylpropyl polystyrene (16, $1.40 \mathrm{mmol}$ of Si per gram, 50-100 mesh; The polymer matrix is copolystyrene-1\% divinylbenzene.) $)^{7,8}$ that had been dried under vacuum for $12 \mathrm{~h}$ was weighed $(212 \mathrm{mg}, 0.297 \mathrm{mmol})$ into a flask and swollen in $\mathrm{CH}_{2} \mathrm{Cl}_{2}(2.1 \mathrm{~mL}, 10 \mathrm{~mL}$ of solvent per gram of resin) under $\mathrm{N}_{2}$ atomosphere for $30 \mathrm{~min}$. The solvent was then drained under positive $\mathrm{N}_{2}$ pressure, and a $4 \%$ trifluoromethanesulfonic acid/ $\mathrm{CH}_{2} \mathrm{Cl}_{2}$ solution (6 equiv of TfOH relative to $\mathrm{Si}$ ) was added by syringe. The resin turned bright red/orange upon acid treatment and was then gently agitated for $30 \mathrm{~min}$ while still under $\mathrm{N}_{2}$ atmosphere. Once activation was completed, two $\mathrm{CH}_{2} \mathrm{Cl}_{2}$ washed removed excess acid. Treatment of silyl triflate functionalized resin with 2,6-lutidine ( $280 \mu \mathrm{L}, 2.40 \mathrm{mmol}, 8$ equiv relative to $\mathrm{Si}$ ) for $15 \mathrm{~min}$ followed by addition 
of an azeotropically dried solution of $1\left(\mathrm{Ar}=2,4,6-i-\mathrm{Pr}_{3} \mathrm{C}_{6} \mathrm{H}_{2}, \mathrm{X}=\mathrm{OH} ; 253 \mathrm{mg}, 0.600 \mathrm{mmol}\right)$ in $\mathrm{CH}_{2} \mathrm{Cl}_{2}(1.2 \mathrm{~mL})$ resulted in a colorless resin. The beads are then gently agitated for an additional $10 \mathrm{~h}$ under $\mathrm{N}_{2}$ atmosphere. The beads were drained, exposed to atmosphere, and subjected to the following wash protocol: $\mathrm{CH}_{2} \mathrm{Cl}_{2}(2 \times 3 \mathrm{~mL} \times 45 \mathrm{~min})$, THF $(2 \times 3 \mathrm{~mL} \times 30 \mathrm{~min})$, $\mathrm{THF} / i-\mathrm{Pr}_{2} \mathrm{EtN}(3: 1,2 \times 3 \mathrm{~mL} \times 30 \mathrm{~min}), \mathrm{THF} / \mathrm{IPA}(3: 1,2 \times 3 \mathrm{~mL} \times 30 \mathrm{~min}), \mathrm{THF} / \mathrm{H}_{2} \mathrm{O}(3: 1,2 \times 3$ $\mathrm{mL} \times 30 \mathrm{~min})$, and THF/IPA (3:1,2 × $3 \mathrm{~mL} \times 30 \mathrm{~min}), \mathrm{DMF}(2 \times 3 \mathrm{~mL} \times 30 \mathrm{~min}), \mathrm{THF}(2 \times 3 \mathrm{~mL} \times$ $30 \mathrm{~min}$ ). The resin was air-dried for $3 \mathrm{~h}$ and then placed under high vacuum for $24 \mathrm{~h}$ to remove trace solvent and $\mathrm{H}_{2} \mathrm{O}$ to give 2 . The mass of 2 was $278 \mathrm{mg}(0.229 \mathrm{mmol}, 0.824 \mathrm{mmol}$ of imidazole moiety per gram), indicating an apparent loading efficiency of $77 \%$ based on weight gain.

Procedure for the Kinetic Resolution of ( \pm )-6c Induced by Reusable Catalyst 2. To a suspension of $( \pm)-6 \mathbf{c}(53.3 \mathrm{mg}, 0.25 \mathrm{mmol})$ and $2(15.2 \mathrm{mg}, 0.0125 \mathrm{mmol}, 0.824 \mathrm{mmol} / \mathrm{g})$ in $\mathrm{CCl}_{4}$ $(2.5 \mathrm{~mL})$ was added $i$ - $\operatorname{Pr}_{2} \mathrm{NEt}(21.8 \mu \mathrm{L}, 0.125 \mathrm{mmol})$ and isobutyric anhydride $(20.7 \mu \mathrm{L}, 0.125$ mmol). After being shaken at $0{ }^{\circ} \mathrm{C}$ for $7 \mathrm{~h}, 2$ was recovered by filtration and washing with toluene $(3 \mathrm{~mL} \times 2)$. Thus, 2 was reused more than 6 times without any loss of activity and selectivity. The combined filtrate was concentrated under reduced pressure, and the residue was analyzed without purification. The ee values for the recovered alcohol $\mathbf{6 c}$ and the acylated product 7c were determined by HPLC analysis: $(1 S, 2 R)$-6c (major enantiomer), $82-85 \%$ ee and $(1 R, 2 S)$-7c (major enantiomer), $87-90 \%$ ee. The conversion from $\mathbf{6 c}$ to $\mathbf{7 c}$ was determined to be $48-49 \%$ by the following equation; conversion $(\%)=[$ ee (recovered alcohol) $] /[$ ee $($ recovered alcohol) + ee (acylated product)]. ${ }^{3}$

\section{Computational Methods}

Theoretical calculations were performed using the Gaussian 98 program. ${ }^{10}$ Gradientcorrected density functional theory (DFT) with Becke's three-parameter exchange with the Lee, Yang and Parr correlation functional (B3LYP), ${ }^{10}$ were carried out using the 6-311++G(d,p) basis set. After satisfactory optimization, the vibrational spectrum of each species was calculated.

\section{The Conformers of 3-Acetyl-1,5-dimethylimidazolium Cation}




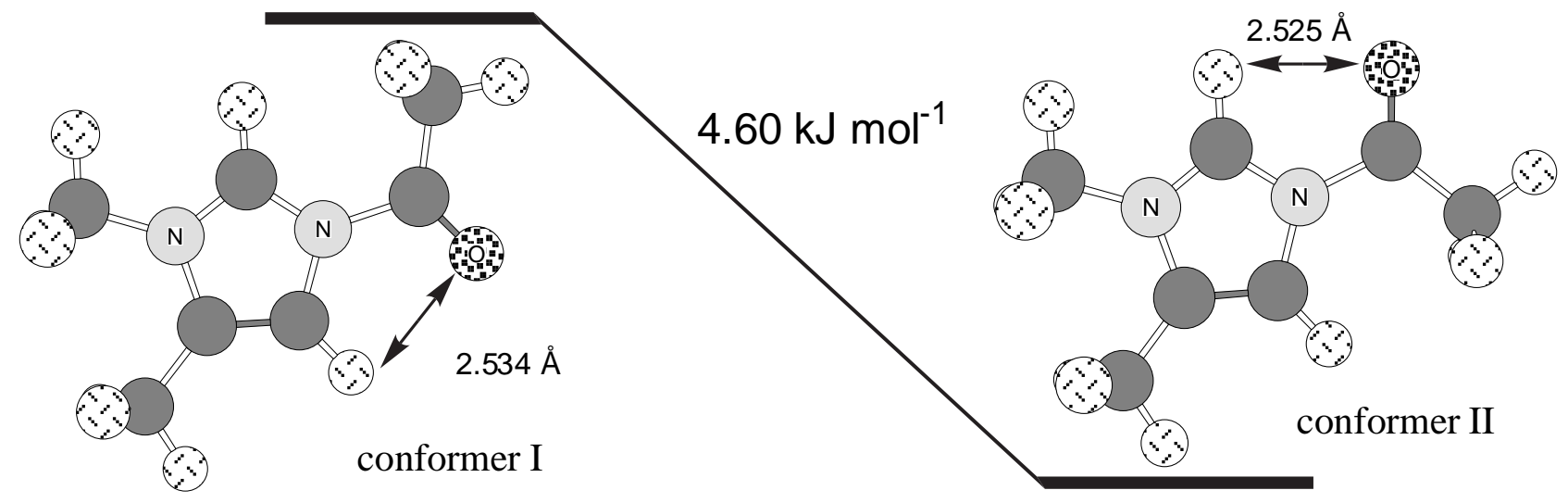

Chart 1.

As shown above, the energy differences between two optimized conformers of 3-acetyl-1,5dimethylimidazolium cation were calculated at the B3LYP/6-311++G(d,p) level. The calculations show that the attractive interaction between an acyl oxygen and an imidazoyl proton in conformer II is stronger than that in conformer I.

\section{Full Molecules}

DMAP

B3LYP / 6-311++G $(\mathrm{d}, \mathrm{p})$

\begin{tabular}{|c|c|c|c|c|c|}
\hline Center & Atomic & Atomic & & es & (Angstroms) \\
\hline Number & Number & Type & $\mathrm{X}$ & Y & Z \\
\hline
\end{tabular}

$\begin{array}{cccccc}1 & 6 & 0 & 0.179133 & -0.000011 & -0.049187 \\ 2 & 7 & 0 & 1.554736 & -0.000004 & -0.108986 \\ 3 & 6 & 0 & -0.571897 & 1.195864 & -0.019946 \\ 4 & 6 & 0 & 2.284689 & 1.249997 & 0.041342 \\ 5 & 6 & 0 & 2.284728 & -1.249983 & 0.041337 \\ 6 & 6 & 0 & -0.571913 & -1.195880 & -0.019942 \\ 7 & 6 & 0 & -1.958056 & 1.131369 & 0.012683 \\ 8 & 6 & 0 & -1.958071 & -1.131367 & 0.012684 \\ 9 & 7 & 0 & -2.671936 & 0.000006 & 0.027327 \\ 10 & 1 & 0 & -0.093789 & 2.165334 & -0.021143 \\ 11 & 1 & 0 & 3.347677 & 1.059383 & -0.099546 \\ 12 & 1 & 0 & 2.143995 & 1.703243 & 1.032449 \\ 13 & 1 & 0 & 1.976366 & 1.977225 & -0.714769\end{array}$




$\begin{array}{llllll}14 & 1 & 0 & 3.347712 & -1.059329 & -0.099526 \\ 15 & 1 & 0 & 1.976451 & -1.977211 & -0.714792 \\ 16 & 1 & 0 & 2.144031 & -1.703249 & 1.032434 \\ 17 & 1 & 0 & -0.093820 & -2.165356 & -0.021131 \\ 18 & 1 & 0 & -2.529941 & 2.055731 & 0.031901 \\ 19 & 1 & 0 & -2.529968 & -2.055721 & 0.031910\end{array}$

$E(B 3 L Y P)=-382.3601616$

Dipole moment (Debye): $X=4.8342 \quad Y=0.0000 \quad Z=\quad 0.1015$ Tot $=\quad 4.8352$

$1,5-\mathrm{Me}_{2}-\mathrm{IMD}$

B3LYP / 6-311++G $(d, p)$

\begin{tabular}{|c|c|c|c|c|c|}
\hline \multirow{2}{*}{$\begin{array}{l}\text { Center } \\
\text { Number }\end{array}$} & \multirow{2}{*}{$\begin{array}{l}\text { Atomic } \\
\text { Number }\end{array}$} & \multirow{2}{*}{$\begin{array}{l}\text { Atomic } \\
\text { Type }\end{array}$} & \multicolumn{3}{|c|}{ Coordinates } \\
\hline & & & $\mathrm{x}$ & $\mathrm{Y}$ & $\mathrm{Z}$ \\
\hline 1 & 6 & 0 & 1.236072 & 0.896166 & 0.000037 \\
\hline 2 & 7 & 0 & -0.116205 & 0.676185 & -0.000289 \\
\hline 3 & 7 & 0 & 1.920180 & -0.222594 & -0.000543 \\
\hline 4 & 6 & 0 & -0.297102 & -0.698820 & 0.000058 \\
\hline 5 & 6 & 0 & -1.155773 & 1.690398 & 0.000189 \\
\hline 6 & 6 & 0 & 0.974292 & -1.221118 & 0.000205 \\
\hline 7 & 6 & 0 & -1.639907 & -1.348816 & 0.000207 \\
\hline 8 & 1 & 0 & 1.649941 & 1.894066 & -0.000125 \\
\hline 9 & 1 & 0 & -1.787836 & 1.604638 & -0.887232 \\
\hline 10 & 1 & 0 & -0.686145 & 2.673846 & -0.002958 \\
\hline 11 & 1 & 0 & -1.783715 & 1.608246 & 0.890907 \\
\hline 12 & 1 & 0 & 1.254033 & -2.263958 & 0.000319 \\
\hline 13 & 1 & 0 & -1.518142 & -2.432937 & 0.000462 \\
\hline 14 & 1 & 0 & -2.230733 & -1.082735 & 0.883833 \\
\hline 15 & 1 & 0 & -2.230716 & -1.083162 & -0.883563 \\
\hline
\end{tabular}

$E(B 3 L Y P)=-304.9316185$

Dipole moment (Debye): $\mathrm{X}=-4.1501 \quad \mathrm{Y}=1.6432 \quad \mathrm{Z}=\mathbf{0} .0012$ 
1-Me-IMD

B3LYP / 6-311++G (d, p)

\begin{tabular}{|c|c|c|c|c|c|}
\hline \multirow{2}{*}{$\begin{array}{l}\text { Center } \\
\text { Number }\end{array}$} & \multirow{2}{*}{$\begin{array}{l}\text { Atomic } \\
\text { Number }\end{array}$} & \multirow{2}{*}{$\begin{array}{l}\text { Atomic } \\
\text { Type }\end{array}$} & \multicolumn{3}{|c|}{ Coordinates (Angstroms) } \\
\hline & & & $\mathrm{X}$ & $\mathrm{Y}$ & Z \\
\hline 1 & 6 & 0 & -0.198976 & -1.085408 & 0.000186 \\
\hline 2 & 7 & 0 & 0.609891 & 0.016246 & -0.000009 \\
\hline 3 & 7 & 0 & -1.473865 & -0.767781 & -0.000197 \\
\hline 4 & 6 & 0 & -0.227521 & 1.113342 & -0.000179 \\
\hline 5 & 6 & 0 & 2.063356 & 0.031762 & 0.000013 \\
\hline 6 & 6 & 0 & -1.502442 & 0.606560 & 0.000182 \\
\hline 7 & 1 & 0 & 0.203649 & -2.087586 & 0.000246 \\
\hline 8 & 1 & 0 & 0.153600 & 2.121246 & -0.000330 \\
\hline 9 & 1 & 0 & 2.445375 & 0.536900 & -0.889970 \\
\hline 10 & 1 & 0 & 2.426754 & -0.995600 & -0.000053 \\
\hline 11 & 1 & 0 & 2.445347 & 0.536780 & 0.890077 \\
\hline 12 & 1 & 0 & -2.433408 & 1.151474 & 0.000264 \\
\hline
\end{tabular}

$E(B 3 L Y P)=-265.6019855$

Dipole moment (Debye): $X=4.0735 \quad \mathrm{Y}=1.2684 \quad \mathrm{Z}=\quad 0.0004$ Tot $=\quad 4.2664$

$1,4-\mathrm{Me}_{2}-\mathrm{IMD}$

B3LYP / 6-311++G $(d, p)$

\begin{tabular}{cccccc} 
Center & Atomic & Atomic & \multicolumn{2}{c}{ Coordinates } \\
Number & Number & Type & $\mathrm{X}$ & $\mathrm{Y}$ & $\mathrm{Z}$ \\
$\mathbf{1}$ & 6 & 0 & -0.608683 & 1.188791 & -0.000077 \\
2 & 7 & 0 & -1.087221 & -0.088539 & -0.000152 \\
3 & 7 & 0 & 0.704603 & 1.228655 & 0.000085
\end{tabular}




$\begin{array}{cccccc}4 & 6 & 0 & 0.021316 & -0.915229 & -0.000034 \\ 5 & 6 & 0 & -2.479709 & -0.501383 & -0.000300 \\ 6 & 6 & 0 & 1.117975 & -0.086793 & 0.000148 \\ 7 & 6 & 0 & 2.566798 & -0.453471 & 0.000348 \\ 8 & 1 & 0 & -1.268080 & 2.044569 & -0.000152 \\ 9 & 1 & 0 & -0.070891 & -1.989239 & -0.000067 \\ 10 & 1 & 0 & -2.709471 & -1.092418 & -0.890158 \\ 11 & 1 & 0 & -3.111359 & 0.386925 & -0.000479 \\ 12 & 1 & 0 & -2.709723 & -1.092243 & 0.889610 \\ 13 & 1 & 0 & 3.071624 & -0.046156 & -0.880308 \\ 14 & 1 & 0 & 2.698653 & -1.537648 & 0.000404 \\ 15 & 1 & 0 & 3.071396 & -0.046090 & 0.881105\end{array}$

$E(B 3 L Y P)=-304.9328651$

Dipole moment (Debye): $\mathrm{X}=-4.1501 \quad \mathrm{Y}=1.6432 \quad \mathrm{Z}=\quad 0.0012$ Tot $=4.4635$

$1,2-\mathrm{Me}_{2}-\mathrm{IMD}$

B $3 L Y P / 6-311++G(d, p)$

\begin{tabular}{|c|c|c|c|c|}
\hline Center & Atomic & Atomic & Coordinates & (Angstroms) \\
\hline Number & Number & Type & $\mathrm{x}$ & Z \\
\hline
\end{tabular}

$\begin{array}{cccccc}1 & 6 & 0 & -0.256975 & -0.688276 & -0.003807 \\ 2 & 7 & 0 & -0.135804 & 0.679893 & -0.022385 \\ 4 & 7 & 0 & 0.925605 & -1.271328 & 0.010330 \\ 5 & 6 & 0 & 1.218440 & 0.961976 & -0.008571 \\ 6 & 6 & 0 & -1.213901 & 1.654620 & 0.015091 \\ 7 & 6 & 0 & 1.849112 & -0.252483 & 0.008020 \\ 8 & 6 & 0 & -1.569775 & -1.398654 & -0.003175 \\ 9 & 1 & 0 & 1.588050 & 1.974218 & -0.018017 \\ 10 & 1 & 0 & -0.832899 & 2.621586 & -0.312600 \\ 11 & 1 & 0 & -1.621013 & 1.761471 & 1.024936 \\ 12 & 1 & 0 & -2.018130 & 1.355938 & -0.659047 \\ & 1 & 0 & 2.909563 & -0.451293 & 0.013538\end{array}$




\begin{tabular}{|c|c|c|c|c|c|}
\hline 13 & 1 & 0 & -2.178337 & -1.140578 & 0.870053 \\
\hline 14 & 1 & 0 & -1.376595 & -2.470271 & 0.018012 \\
\hline 15 & 1 & 0 & -2.160642 & -1.174123 & -0.897846 \\
\hline
\end{tabular}

$E(B 3 L Y P)=-304.9334031$

Dipole moment (Debye): $X=-2.5782 \quad Y=\quad 3.2048 \quad Z=\quad-0.0103$ Tot $=\quad 4.1132$

3-acetyl-1,5-dimethylimidazolium cation (conformer I; Chart 1.) B 3LYP / 6-311++G (d,p)

\begin{tabular}{|c|c|c|c|c|c|}
\hline \multirow{2}{*}{$\begin{array}{l}\text { Center } \\
\text { Number }\end{array}$} & \multirow{2}{*}{$\begin{array}{l}\text { Atomic } \\
\text { Number }\end{array}$} & \multirow{2}{*}{$\begin{array}{l}\text { Atomic } \\
\text { Type }\end{array}$} & \multicolumn{3}{|c|}{ Coordinates (Angstroms) } \\
\hline & & & $\mathrm{x}$ & Y & Z \\
\hline 1 & 6 & 0 & -0.027811 & 1.040098 & -0.000323 \\
\hline 2 & 7 & 0 & -1.313389 & 0.696498 & -0.000047 \\
\hline 3 & 7 & 0 & 0.714366 & -0.080551 & -0.000024 \\
\hline 4 & 6 & 0 & -1.417998 & -0.701657 & -0.000034 \\
\hline 5 & 6 & 0 & -2.443153 & 1.636793 & 0.000146 \\
\hline 6 & 6 & 0 & 2.192882 & -0.212847 & 0.000065 \\
\hline 7 & 6 & 0 & -0.141662 & -1.173824 & -0.000038 \\
\hline 8 & 8 & 0 & 2.628832 & -1.319177 & 0.000286 \\
\hline 9 & 6 & 0 & 2.961631 & 1.072438 & -0.000024 \\
\hline 10 & 6 & 0 & -2.721368 & -1.420507 & -0.000048 \\
\hline 11 & 1 & 0 & 0.346306 & 2.049679 & -0.000483 \\
\hline 12 & 1 & 0 & -3.049548 & 1.478666 & -0.891241 \\
\hline 13 & 1 & 0 & -2.056754 & 2.653933 & -0.000824 \\
\hline 14 & 1 & 0 & -3.048347 & 1.479878 & 0.892571 \\
\hline 15 & 1 & 0 & 0.245338 & -2.178366 & 0.000014 \\
\hline 16 & 1 & 0 & 4.022393 & 0.830931 & 0.000040 \\
\hline 17 & 1 & 0 & 2.730918 & 1.667133 & 0.888370 \\
\hline 18 & 1 & 0 & 2.730983 & 1.666981 & -0.888536 \\
\hline 19 & 1 & 0 & -2.544491 & -2.495614 & 0.000752 \\
\hline 20 & 1 & 0 & -3.315064 & -1.176591 & 0.885183 \\
\hline 21 & 1 & 0 & -3.314350 & -1.177803 & -0.886099 \\
\hline
\end{tabular}

\section{S16}


$E(B 3 L Y P)=-458.0035914$

3-acetyl-1,5-dimethylimidazolium cation (conformer II; Chart 1.) B $3 L Y P / 6-311++G(d, p)$

\begin{tabular}{|c|c|c|c|c|c|}
\hline \multirow{2}{*}{$\begin{array}{l}\text { Center } \\
\text { Number }\end{array}$} & \multirow{2}{*}{$\begin{array}{l}\text { Atomic } \\
\text { Number }\end{array}$} & \multirow{2}{*}{$\begin{array}{l}\text { Atomic } \\
\text { Type }\end{array}$} & \multicolumn{3}{|c|}{ Coordinates (Angstroms) } \\
\hline & & & $\mathrm{x}$ & $\mathrm{Y}$ & Z \\
\hline 1 & 6 & 0 & 0.108588 & -1.082678 & -0.000215 \\
\hline 2 & 7 & 0 & 1.362366 & -0.643507 & -0.000056 \\
\hline 3 & 7 & 0 & -0.717857 & -0.023848 & -0.000022 \\
\hline 4 & 6 & 0 & 1.363460 & 0.758480 & 0.000010 \\
\hline 5 & 6 & 0 & 2.558362 & -1.498730 & -0.000034 \\
\hline 6 & 6 & 0 & -2.193442 & -0.188283 & 0.000024 \\
\hline 7 & 6 & 0 & 0.054500 & 1.133488 & 0.000113 \\
\hline 8 & 8 & 0 & -2.609820 & -1.303980 & -0.000011 \\
\hline 9 & 6 & 0 & -2.977536 & 1.084007 & 0.000155 \\
\hline 10 & 6 & 0 & 2.610723 & 1.571109 & -0.000015 \\
\hline 11 & 1 & 0 & -0.217087 & -2.110084 & -0.000356 \\
\hline 12 & 1 & 0 & 3.151005 & -1.297187 & -0.891905 \\
\hline 13 & 1 & 0 & 2.244840 & -2.540483 & -0.000620 \\
\hline 14 & 1 & 0 & 3.150440 & -1.298007 & 0.892401 \\
\hline 15 & 1 & 0 & -0.378510 & 2.118098 & 0.000259 \\
\hline 16 & 1 & 0 & -4.035748 & 0.832081 & -0.000355 \\
\hline 17 & 1 & 0 & -2.746991 & 1.682295 & -0.885914 \\
\hline 18 & 1 & 0 & -2.747719 & 1.681506 & 0.886961 \\
\hline 19 & 1 & 0 & 2.358974 & 2.631245 & 0.000685 \\
\hline 20 & 1 & 0 & 3.220276 & 1.369248 & 0.884959 \\
\hline 21 & 1 & 0 & 3.219578 & 1.370255 & -0.885707 \\
\hline
\end{tabular}

$E(B 3 L Y P)=-458.0053486$ 


\section{References}

1. Gonzalez, F. B.; Baz, J. P.; Espina, M. I. Tetrahedron Lett. 1989, 30, 2145.

2. Matsumoto, K.; Sato, Y.; Shimojo, M.; Hatanaka, M. Tetrahedron: Asymmetry 2000, 11, 1965.

3. Kagan, H. B.; Fiaud, J. C. Top. Stereochem. 1988, 18, 249.

4. Kawabata, T.; Nagato, M.; Takasu, K.; Fuji, K. J. Am. Chem. Soc. 1997, 119, 3169.

5. Tomio, K.; Kawabata, T.; Nagato, M. Jpn. Kokai Tokkyo Koho 1998, JP 10077283, A2 19980324Heisei, 9 pp.

6. (a) Kawabata, T.; Yamamoto, K.; Momose, Y.; Yoshida, H.; Nagaoka, Y.; Fuji, K. Chem. Commun. 2001, 2700. (b) Priem, G.; Pelotier, B.; Macdonald, S. J. F.; Anson, M. S.; Campbell, I. B. J. Org. Chem. 2003, 68, 3844.

7. (a) Woodlard, F. X.; Paetsch, J.; Ellman, J. A. J. Org. Chem. 1997, 62, 6102. (b) Tallarico, J. A.; Depew, K. M.; Pelish, H. E.; Westwood, N. J.; Lindsley, C. W.; Shair, M. D.; Schreiber, S. L.; Foley, M. A. J. Comb. Chem. 2001, 3, 312.

8. 16 was purchased from Novabiochem ${ }^{\circledR}$.

9. Frisch, M. J.; Trucks, G. W.; Schlegel, H. B.; Scuseria, G. E.; Robb, M. A.; Cheeseman, J. R.; Zakrzewski, V. G.; Montgomery, J. A., Jr.; Stratmann, R. E.; Burant, J. C.; Dapprich, S.; Millam, J. M.; Daniels, A. D.; Kudin, K. N.; Strain, M. C.; Farkas, O.; Tomasi, J.; Barone, V.; Cossi, M.; Cammi, R.; Mennucci, B.; Pomelli, C.; Adamo, C.; Clifford, S.; Ochterski, J.; Petersson, G. A.; Ayala, P. Y.; Cui, Q.; Morokuma, K.; Malick, D. K.; Rabuck, A. D.; Raghavachari, K.; Foresman, J. B.; Cioslowski, J.; Ortiz, J. V.; Stefanov, B. B.; Liu, G.; Liashenko, A.; Piskorz, P.; Komaromi, I.; Gomperts, R.; Martin, R. L.; Fox, D. J.; Keith, T.; Al-Laham, M. A.; Peng, C. Y.; Nanayakkara, A.; Gonzalez, C.; Challacombe, M.; Gill, P. M. W.; Johnson, B.; Chen, W.; Wong, M. W.; Andres, J. L.; Gonzalez, C.; Head-Gordon, M.; Replogle, E. S.; Pople, J. A. Gaussian 98; Gaussian, Inc.: Pittsburgh, PA, 1998.

10. (a) Becke, A. D. J. Chem. Phys. 1993, 96, 5648. (b) Stevens, P. J.; Devlin, J. F.; Chablowski, C. F.; Frisch, M. J. J. Phys. Chem. 1994, 98, 11623. 


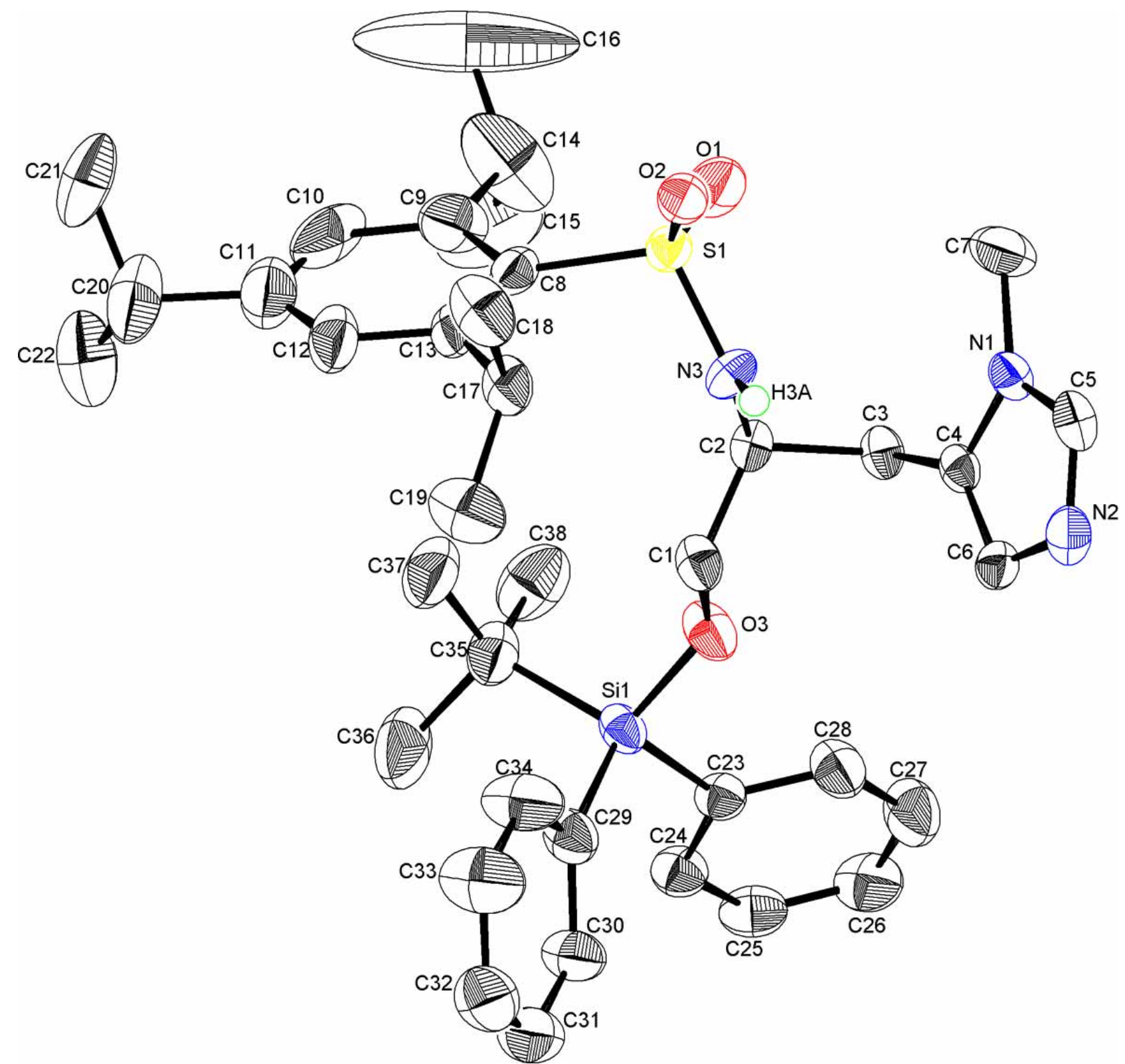

Figure. ORTEP Plot of 1d. Drawn with 50\% probability, and hydrogen atoms except for the $\mathrm{SO}_{2} \mathrm{NH}$ moiety are omitted for clarity. 